\title{
Differential Commuting Operator and Closed-Form Eigenfunctions for Linear Canonical Transforms
}

\author{
Soo-Chang $\mathrm{Pei}^{1, *}$ and Chun-Lin $\mathrm{Liu}^{2}$ \\ ${ }^{1,2}$ Department of Electrical Engineering, National Taiwan University, No. 1, Sec. 4, \\ Roosevelt Road, Taipei 10617, Taiwan \\ *Corresponding author: pei@cc.ee.ntu.edu.tw
}

The linear canonical transform (LCT) with $a, b, c, d$ parameter plays an important role in quantum mechanics, optics, and signal processing. The eigenfunctions of the LCT are also important because they describe the self-imaging phenomenon in optical systems. However, the existing solutions for the eigenfunctions of the LCT are divided into many cases and they lack a systematic way to solve these eigenfunctions. In this paper, we find a linear, second-order, self-adjoint differential commuting operator that commutes with the linear canonical transform operator. Hence, the commuting operator and the LCT share the same eigenfunctions with different eigenvalues. The commuting operator is very general and simple when it is compared to the existing multiple-parameter differential equations. Then, the eigenfunctions can be derived systematically. The eigenvalues of the commuting operator have closed-form relationships with the eigenvalues of the LCT. We also simplify the eigenfunctions for $|a+d|>2$ and $a+d= \pm 2, b \neq 0$ into the more compact closed form instead of the integral form. For $|a+d|>2$, the eigenfunctions are related to the parabolic cylinder functions. (c) 2013 Optical Society of America

OCIS codes: 070.0070, 070.2580, 070.2590, 070.2575 .

\section{Introduction}

Linear canonical transform (LCT) is a phase space integral transform that is a function of several real parameters, $a, b, c$, and $d$, where $a d-b c=1$. LCT is a generalization of Fourier transform, fractional Fourier transform (FrFT), Fresnel transform, scaling operation, and chirp multiplication operation [1-3]. It is also called canonical transforms [3], generalized 
Fresnel transforms [4], ABCD transforms [5], or quadratic-phase integrals [1]. The LCT finds useful applications in quantum mechanics, optics, digital holography, and signal processing. In quantum mechanics, FrFT can be used for solving the time-dependent Schrödinger equation $[3,6,7]$. The first-order optical systems can be analyzed by LCT $[1,5,8]$. Speckle metrology systems and spatial coherence were designed and analyzed by Yura et al. using the LCT $[9,10]$. In digital holography, the LCT finds application in understanding the filtering role of CCD pixels and finite apertures [11]. LCT also twists the time-frequency representation of the signal so we can perform time-frequency analysis [1] and filter design [12]. A full study on the fractional Fourier transform and the Wigner distribution function is referred to $[13]$.

The eigenfunctions of LCT have been known for many years. In [4], the eigenfunctions of LCT were studied. The closed-form solutions are in the form of scaled Hermite Gaussian functions multiplied by the chirp functions [4]. However, the solutions are valid only when $\mid a+$ $d \mid<2$. In $[14,15]$, the eigenfunctions for all cases were discussed. However, the eigenfunctions for different cases are quite different. It seems that there are no general-forms for all these distinct eigenfunctions. In addition, some eigenfunctions are expressed in integral forms. For instance, when $|a+d|>2$, the solution is related to the self-similar functions, or fractals [16]. There is no closed-form solution for these self-similar functions so The final eigenfunction is described as integrals. Recently, the eigenfunction of the LCT for $|a+d|>2$ was derived to be a complex-scaled Hermite Gaussian function with a chirp term, which is unbounded $[17,18]$.

In this paper, we take a different point of view on the eigenfunctions and then complete the study of eigenfunctions in [14]. We find a linear operator $\mathcal{C}_{\mathbf{M}}$ which commutes with LCT operator. As a result, $\mathcal{C}_{\mathbf{M}}$ and LCT operator share common eigenfunctions with different eigenvalues. This operator is the linear combination of $\mathcal{D}_{t}^{2}, t \mathcal{D}_{t}+\mathcal{D}_{t} t$, and $t^{2}$ with proper combination coefficients, where $\mathcal{D}_{t}=\mathrm{d} / \mathrm{d} t$ is the differential operator. This commuting operator always has the same general form with arbitrary $a, b, c$, and $d$ parameters. However, the eigenvalues of $\mathcal{C}_{\mathbf{M}}$ vary for different $a, b, c$, and $d$. We show that the eigenvalues of $\mathcal{C}_{\mathbf{M}}$ are related to the eigenvalues of LCT by certain formulae. The operator $\mathcal{C}_{\mathbf{M}}$ is shown to be a self-adjoint operator so that the eigenfunctions are orthonormal, whatever the parameters $a, b, c$, and $d$ are. With the help of $\mathcal{C}_{\mathbf{M}}$, we complete the study of the eigenfunctions in [14]. Notably, for $|a+d|>2$, the solution can be expressed in terms of the parabolic cylinder functions, which are generalizations of the Hermite Gaussian eigenfunctions in the $|a+d|<2$ case.

This paper is summarized as follows. First, in Section 2, LCT and its eigenfunctions are briefly reviewed for clarity. The linear operator $\mathcal{C}_{\mathbf{M}}$ is defined and its many properties are investigated in Section 3. In Section 4, the complete study on the eigenfunctions of LCT is given based on both $\mathcal{C}_{\mathbf{M}}$ and the previous results. We prove that our results are consistent 
with the previous work and, in addition, these eigenfunctions are not only with closed forms but are also orthonormal. In Section 5, we discuss the symmetry relations among the all eigenvalues and eigenfunctions and give a short example to explain the physical meaning of the solution. A comparison is made to distinguish our eigenfunctions for $a+d>2$ and those in $[17,18]$. Section 6 points out some signal processing applications of the eigenfunctions of the LCT, including an illustrative example to clarify the role of eigenfunction in implementing the LCT. Finally, in Section 7, we summarize the result and make a conclusion.

\section{Preliminary}

\section{A. Linear Canonical Transforms}

LCT is defined for $b \neq 0$ by the following integral

$$
\begin{aligned}
& X_{\mathbf{M}}(u)=\left(\mathcal{L}_{\mathbf{M}} x\right)(u) \\
& =\sqrt{\frac{1}{j 2 \pi b}} \int_{-\infty}^{\infty} x(t) e^{j \frac{a}{2 b} t^{2}} e^{-j \frac{1}{b} t u} e^{j \frac{d}{2 b} u^{2}} \mathrm{~d} t, b \neq 0, t, u \in \mathbb{R} .
\end{aligned}
$$

The LCT can also be regarded as a transformation mapping from $x$ to $X_{\mathbf{M}}$. In this paper, we write the transformed result as $X_{\mathbf{M}}(t)$ to facilitate the discussion about the operators. When $b=0$, the definition is modified into

$$
X_{\mathbf{M}}(t)=\sqrt{d} e^{j \frac{c d}{2} t^{2}} x(t d), b=0
$$

The 2 -by-2 matrix $\mathbf{M}=[a, b ; c, d]$ is a real-valued matrix with $\operatorname{det}(\mathbf{M})=1$. The operator $\mathcal{L}_{\mathbf{M}}$, or sometimes denoted by $\mathcal{L}_{a, b, c, d}$, is the linear canonical transform operator. The degree of freedom of LCT is three with the constraint $\operatorname{det}(\mathbf{M})=1$.

The LCT has the additive property and the reversible property.

$$
\mathcal{L}_{\mathbf{M}_{1} \mathbf{M}_{2}}=\mathcal{L}_{\mathbf{M}_{1}} \mathcal{L}_{\mathbf{M}_{2}}, \quad \mathcal{L}_{\mathbf{M}}^{-1}=\mathcal{L}_{\mathbf{M}^{-1}}
$$

These properties enable us to decompose LCT with arbitrary $\mathbf{M}$ into the product of some basic elementary operations [19]. Implementing these basic operations step by step gives us the final result.

These basic operations are all special cases of LCT. They are summarized as follows:

1. Fourier transform (FT) corresponds the LCT with $\mathbf{M}=[0,1 ;-1,0]$. Note that there is an extra phase term $\sqrt{j}$ in front of the conventional Fourier transform.

2. Fractional Fourier transform (FrFT) with fractional order $\alpha \in[0,2 \pi)[1,7,15]$ is the LCT with $\mathbf{M}=[\cos \alpha, \sin \alpha ;-\sin \alpha, \cos \alpha]$, still with the constant phase term, $\sqrt{j}$. Our definition is consistent with that in [15]. 
3. Fresnel transform is obtained from the LCT with $\mathbf{M}=[1, b ; 0,1]$. In optics, Fresnel transform describes the wave propagation in free space [20]. In signal processing or time-frequency analysis, this case is also called chirp convolution operation.

4. Scaling operation corresponds the LCT with $\mathbf{M}=\left[\sigma^{-1}, 0 ; 0, \sigma\right]$. From (2), the scaling operation is then $\sqrt{\sigma} x(\sigma t)$.

5. Chirp multiplication operation is derived from the LCT with $\mathbf{M}=[1,0 ; c, 1]$. In this case, the operation is $e^{j \frac{c}{2} t^{2}} x(t)$.

All of the relationships and the details about these special transforms can be found in $[1,3]$.

The LCT has lots of mathematical properties. We want to note the multiplication-by- $t$ property and the derivative property specifically (see Chapter 2 in [15] and Section 3.4 in [1]), which are

$$
\begin{aligned}
\mathcal{L}_{\mathbf{M}}\{t x(t)\} & =\left(j b \frac{\mathrm{d}}{\mathrm{d} t}+t d\right) X_{\mathbf{M}}(t), \\
\mathcal{L}_{\mathbf{M}}\left\{\frac{\mathrm{d}}{\mathrm{d} t} x(t)\right\} & =\left(a \frac{\mathrm{d}}{\mathrm{d} t}-j c t\right) X_{\mathbf{M}}(t),
\end{aligned}
$$

where the left-hand side $x(t)$ denotes the original signal and the right-hand side $X_{\mathbf{M}}(t)$ stands for its LCT. (4) and (5) can be further rewritten as the operator form by

$$
\begin{aligned}
\mathcal{L}_{\mathbf{M}} t & =\left(j b \mathcal{D}_{t}+t d\right) \mathcal{L}_{\mathbf{M}}, \\
\mathcal{L}_{\mathbf{M}} \mathcal{D}_{t} & =\left(a \mathcal{D}_{t}-j c t\right) \mathcal{L}_{\mathbf{M}} .
\end{aligned}
$$

These two identities will be used throughout this paper. For other LCT properties, we refer to the reader to $[1]$.

\section{B. Previous Work on the Eigenfunctions of $L C T$}

The eigenfunctions and the eigenvalues of LCT were studied very extensively in [14]. They are divided into seven cases and summarized in Table 1.

The eigenfunctions are first divided by the value of $a+d$ and then by the value of $b$. In Case (A) for $|a+d|<2$, the solution is the product of the scaled Hermite Gaussian function and the chirp function, as indicated in the Case (A) of Table 1. In this case, the eigenfunctions form a complete and orthonormal basis for $L^{2}(\mathbb{R})[4]$, where $L^{2}(\mathbb{R})=\left\{\left.x(t)\left|\int_{-\infty}^{\infty}\right| x(t)\right|^{2} \mathrm{~d} t<\infty\right\}$ is the set of finite-energy functions. Hence the eigenfunctions are very suitable for several applications in optics [4].

In Case (B) and Case (C), the eigenfunctions are impulse trains with the amplitude $A_{n}$ for the right-shifted impulses, and with the amplitude $B_{m}$ for the left-shifted impulses. In Case (D) and Case (E), the function $g(t)$ is the linear combination of two complex exponential 
functions but $g(t)$ is not the eigenfunction. The actual eigenfunction is an integral associated with $g(t)$. In addition, in Case $(\mathrm{C})$ and Case (E), the combination coefficients satisfy $A_{n}=$ $\pm B_{n}$ or $C_{n}= \pm D_{n}$.

In Case (F), the eigenfunction is connected with the function $h(t)$, which is the self-similar function, or fractals. The closed-form $h(t)$ and the corresponding eigenvalue $\lambda$ are unknown and not given. As a result, the eigenfunction is left as an open problem to solve. Case $(G)$ limits $h(t)$ to be even or odd functions and the rest part is similar to Case (F).

The existing results still require to be elaborated in terms of the eigenfunctions, eigenvalues, and their properties. First of all, the eigenfunctions can be further simplified into closed forms instead of being represented in integral forms. To do so, it is possible to find the closed-form expression of self-similar signals and then evaluate the integral in Case (D) and Case (F). Secondly, in Case (F), if $b=0$, we have $\eta=0$ and the chirp function in the integral becomes undefined. It is more likely to discuss this special case separately. Finally, because we only discuss the real-parameter LCT in this paper, then $\mathcal{L}_{\mathbf{M}}$ is an unitary operator, which implies that all the eigenfunctions are orthogonal. However, in the existing literature, the orthogonality of the eigenfunctions is not addressed, except for Case (A).

In [18], for $|a+d|>2$, the complex-scaled Hermite Gaussian function is the eigenfunction,

$$
\Psi_{n}^{(\sigma, \tau)}(u)=\frac{1}{\sqrt{\sigma 2^{n} n ! \sqrt{j \pi}}} H_{n}\left(\frac{u}{\sigma e^{j \frac{\pi}{4}}}\right) e^{j \frac{1-\tau}{2 \sigma^{2}} u^{2}}
$$

However, the eigenfunction is not bounded as $u$ approaches infinity, $\left|\Psi_{n}^{(\sigma, \tau)}(u)\right| \rightarrow \infty$ as $u \rightarrow$ $\infty$. This divergent property prevents the eigen-decomposition of the LCT. Our goal is to find a bounded solution in this case and compare the both solutions.

\section{General commuting operator $\mathcal{C}_{\mathrm{M}}$}

In $[1,3]$, the commuting operators for some special cases of the LCT are listed in Table 2. These operators commute with LCT operator for different cases. The commuting operator and LCT share the common eigenfunctions with different eigenvalues. Hence, we can solve the commuting operator to have the eigenfunctions of LCT. This technique has been widely used in the study of the eigenfunctions of discrete Fourier transforms [21,22].

However, the conventional commuting operators in Table 2 are not suitable for the general LCT with arbitrary $a, b, c$, and $d$. Our goal is to find a general expression for the commuting operator, so that we can directly obtain the eigenfunctions by solving the general commuting operator. It is observed that the existing commuting operators are the linear combination of the three basic operators: $\mathcal{D}_{t}^{2}, t \mathcal{D}_{t}+\mathcal{D}_{t} t$, and $t^{2}$. Hence, we assume that the general commuting operator is the linear combination of them. The combination coefficients are directly related to the parameters $a, b, c$, and $d$. 
Definition 3.1. The general commuting operator $\mathcal{C}_{\mathbf{M}}$ is defined by

$$
\mathcal{C}_{\mathbf{M}}=b \mathcal{D}_{t}^{2}+j \frac{a-d}{2}\left(t \mathcal{D}_{t}+\mathcal{D}_{t} t\right)+c t^{2}
$$

where $\mathcal{D}_{t}=\mathrm{d} / \mathrm{d} t$ is the differential operator and $\mathbf{M}=[a, b ; c, d] \in \mathbb{R}^{2 \times 2}$ denotes the real matrix associated with LCT.

Proposition 3.1. $\mathcal{C}_{\mathbf{M}}$ commutes with LCT operator $\mathcal{L}_{\mathbf{M}}$.

Proof. Perform LCT operation $\mathcal{L}_{\mathrm{M}}$ on both sides of (9)

$$
\mathcal{L}_{\mathbf{M}} \mathcal{C}_{\mathbf{M}}=b \mathcal{L}_{\mathbf{M}} \mathcal{D}_{t}^{2}+j \frac{a-d}{2} \mathcal{L}_{\mathbf{M}}\left(t \mathcal{D}_{t}+\mathcal{D}_{t} t\right)+c \mathcal{L}_{\mathbf{M}} t^{2}
$$

Then (6) and (7) can be applied many times to obtain

$$
\begin{aligned}
\mathcal{L}_{\mathbf{M}} \mathcal{D}_{t}^{2} & =\left(a \mathcal{D}_{t}-j c t\right)^{2} \mathcal{L}_{\mathbf{M}}, \\
\mathcal{L}_{\mathbf{M}}\left(t \mathcal{D}_{t}\right) & =\left(j b \mathcal{D}_{t}+t d\right)\left(a \mathcal{D}_{t}-j c t\right) \mathcal{L}_{\mathbf{M}}, \\
\mathcal{L}_{\mathbf{M}}\left(\mathcal{D}_{t} t\right) & =\left(a \mathcal{D}_{t}-j c t\right)\left(j b \mathcal{D}_{t}+t d\right) \mathcal{L}_{\mathbf{M}}, \\
\mathcal{L}_{\mathbf{M}} t^{2} & =\left(j b \mathcal{D}_{t}+t d\right)^{2} \mathcal{L}_{\mathbf{M}}
\end{aligned}
$$

Substituting (11), (12), (13), and (14) into (10) yields

$$
\begin{aligned}
& {\left[b\left(a \mathcal{D}_{t}-j c t\right)^{2}+j \frac{a-d}{2}\left(j b \mathcal{D}_{t}+t d\right)\left(a \mathcal{D}_{t}-j c t\right)\right.} \\
& \left.+j \frac{a-d}{2}\left(a \mathcal{D}_{t}-j c t\right)\left(j b \mathcal{D}_{t}+t d\right)+c\left(j b \mathcal{D}_{t}+t d\right)^{2}\right] \mathcal{L}_{\mathbf{M}} \\
& =\left[\left(b a^{2}+j \frac{a-d}{2}(j 2 a b)-c b^{2}\right) \mathcal{D}_{t}^{2}\right. \\
& +\left(-j a b c+j \frac{a-d}{2}(a d+b c)+j b c d\right)\left(t \mathcal{D}_{t}+\mathcal{D}_{t} t\right) \\
& \left.+\left(-b c^{2}+j \frac{a-d}{2}(-j 2 c d)+c d^{2}\right) t^{2}\right] \mathcal{L}_{\mathbf{M}} .
\end{aligned}
$$

Rearranging the coefficients in the parentheses yields

$$
\begin{aligned}
\mathcal{L}_{\mathrm{M}} \mathcal{C}_{\mathbf{M}} & =\left[b(a d-b c) \mathcal{D}_{t}^{2}+j \frac{(a-d)(a d-b c)}{2}\left(t \mathcal{D}_{t}+\mathcal{D}_{t} t\right)\right. \\
& \left.+c(a d-b c) t^{2}\right] \mathcal{L}_{\mathbf{M}}=\mathcal{C}_{\mathbf{M}} \mathcal{L}_{\mathbf{M}}
\end{aligned}
$$

According to $a d-b c=1$, we obtain $\mathcal{L}_{\mathbf{M}} \mathcal{C}_{\mathbf{M}}=\mathcal{C}_{\mathbf{M}} \mathcal{L}_{\mathbf{M}}$, implying that $\mathcal{C}_{\mathbf{M}}$ commutes with $\mathcal{L}_{\mathrm{M}}$.

Proposition 3.2. $\mathcal{C}_{\mathrm{M}}$ is self-adjoint. 
Proof. Assume that $\mathcal{A}^{\dagger}$ and $\mathcal{B}^{\dagger}$ stands for the adjoint operator of the operator $\mathcal{A}$ and $\mathcal{B}$, respectively. The basic operation rules for adjoint operators are

$$
(\mathcal{A}+\mathcal{B})^{\dagger}=\mathcal{A}^{\dagger}+\mathcal{B}^{\dagger}, \quad(\mathcal{A B})^{\dagger}=\mathcal{B}^{\dagger} \mathcal{A}^{\dagger}
$$

In addition, the some useful adjoint operators are

$$
\mathcal{D}_{t}^{\dagger}=-\mathcal{D}_{t}, \quad\left(\mathcal{D}_{t}^{2}\right)^{\dagger}=\mathcal{D}_{t}^{2}, t^{\dagger}=t, a^{\dagger}=a^{*}
$$

where $a$ is a complex number and $*$ denotes complex conjugates. To prove that $\mathcal{C}_{\mathbf{M}}$ is selfadjoint, taking the adjoint operator of $\mathcal{C}_{\mathbf{M}}$ gives us

$$
\begin{aligned}
\mathcal{C}_{\mathbf{M}}^{\dagger} & =\left(b \mathcal{D}_{t}^{2}\right)^{\dagger}+\left[j \frac{a-d}{2}\left(t \mathcal{D}_{t}+\mathcal{D}_{t} t\right)\right]^{\dagger}+\left(c t^{2}\right)^{\dagger} \\
& =\left(\mathcal{D}_{t}^{2}\right)^{\dagger} b^{\dagger}+\left(t \mathcal{D}_{t}+\mathcal{D}_{t} t\right)^{\dagger}\left(j \frac{a-d}{2}\right)^{\dagger}+\left(t^{2}\right)^{\dagger} c^{\dagger}
\end{aligned}
$$

Note that (19) and (20) are due to the basic operation rules (17). The next step is to apply the known adjoint operators, listed in (18), one by one. Therefore, $\mathcal{C}_{\mathbf{M}}$ can be simplified into

$$
\mathcal{C}_{\mathbf{M}}^{\dagger}=b \mathcal{D}_{t}^{2}+\left(-t \mathcal{D}_{t}-\mathcal{D}_{t} t\right)\left(-j \frac{a-d}{2}\right)+c t^{2},
$$

where the parameters $a, b, c$, and $d$ are all real numbers. Rearranging (21) yields $\mathcal{C}_{\mathbf{M}}^{\dagger}=$ $\mathcal{C}_{\mathrm{M}}$

It is concluded from Proposition 3.1 that $\mathcal{C}_{\mathbf{M}}$ and $\mathcal{L}_{\mathbf{M}}$ have a common set of eigenfunctions with different eigenvalues. Assume that the common eigenfunctions are written as $\psi_{\mu_{\mathbf{M}}}(t)$. By definition, we have

$$
\mathcal{C}_{\mathbf{M}} \psi_{\mu_{\mathbf{M}}}(t)=\mu_{\mathbf{M}} \psi_{\mu_{\mathbf{M}}}(t), \quad \mathcal{L}_{\mathbf{M}} \psi_{\mu_{\mathbf{M}}}(t)=\lambda_{\mathbf{M}} \psi_{\mu_{\mathbf{M}}}(t)
$$

where $\mu_{\mathbf{M}}$ is the eigenvalue of $\mathcal{C}_{\mathbf{M}}$ and $\lambda_{\mathbf{M}}$ is the eigenvalue of $\mathcal{L}_{\mathbf{M}}$. It is noted that $\mu_{\mathbf{M}}$ is not the same as $\lambda_{\mathbf{M}}$ because they correspond different operators. We write the subscript $\mu_{\mathbf{M}}$ in the eigenfunctions in order to specify that the eigenfunctions depend on $\mu_{\mathbf{M}}$.

Then, according to Proposition 3.2 and the fact that $\mathcal{L}_{\mathbf{M}}$ is unitary, we have four major properties

Property 1. The eigenvalues $\mu_{\mathbf{M}}$ are all real.

Property 2. The eigenfunctions $\psi_{\mu_{M}}(t)$ are orthogonal to each other.

Property 3. The eigenfunctions $\psi_{\mu_{M}}(t)$ form a complete set. 


\section{Property 4. $\left|\lambda_{\mathrm{M}}\right|=1$.}

Property 1, Property 2, and Property 3 are derived directly from self-adjoint- $\mathcal{C}_{\mathbf{M}}$ Proposition 3.2 (Section 3.3 and Appendix A.6 in [6]). From the perspective of quantum mechanics, the self-adjoint operator $\mathcal{C}_{\mathbf{M}}$ corresponds to the observables while the eigenfunctions $\psi_{\mu_{\mathrm{M}}}(t)$ are related to determinate states. For other physical meaning of Property 1, Property 2, and Property 3, the reader is referred to Chapter 3 and Appendix A.6 of [6].

Property 4 can be verified by taking the $l_{2}$-norm of the second equation of (22),

$$
\left\|\mathcal{L}_{\mathbf{M}} \psi_{\mu_{\mathbf{M}}}(t)\right\|_{2}=\left|\lambda_{\mathbf{M}}\right|\left\|\psi_{\mu_{\mathbf{M}}}(t)\right\|_{2} .
$$

Note that the LCT operator $\mathcal{L}_{\mathbf{M}}$ is unitary, implying $\left\|\mathcal{L}_{\mathbf{M}} \psi_{\mu_{\mathrm{M}}}(t)\right\|_{2}=\left\|\psi_{\mu_{\mathbf{M}}}(t)\right\|_{2}$. Combining those equations result in $\left|\lambda_{\mathbf{M}}\right|=1$ for non-zero eigenfunctions.

These properties should be satisfied for any cases. As a result, we can verify our derived eigenfunctions with these properties.

Finally, the physical meaning of (22) is discussed. The eigen-equation for $\mathcal{C}_{M}$ can be regarded as the differential equation for the eigenfunctions of the LCT $\psi_{\mu_{\mathrm{M}}}(t)$. This equation is similar to the Helmholtz equation in diffraction optics [23,24] or the Schrödinger equation in quantum mechanics [6]. Furthermore, the eigen-equation of $\mathcal{L}_{\mathbf{M}}$ indicates the effect of the LCT working on the eigenfunctions. Due to Proposition 4, a phase shift is added to the eigenfunctions when LCT is applied. This phenomenon corresponds to the eigenmode in diffraction optics $[23,24]$ or the propagator in quantum mechanics [6].

In terms of signal processing, (22) can be interpreted differently. Our goal is to realize the LCT operation $\mathcal{L}_{\mathbf{M}}$ while the auxiliary differential equation associated with $\mathcal{C}_{\mathbf{M}}$ can be straightforwardly solved. After $\psi_{\mu_{\mathrm{M}}}(t)$ and $\mu_{\mathrm{M}}$ are fully established, we can proceed to implement the LCT with known $\psi_{\mu_{\mathbf{M}}}(t)$ and $\lambda_{\mathbf{M}}$. Then the resultant LCT can be used in topics of signal processing application, such as time-frequency analysis, filtering, signal representation, and signal analysis.

\section{LCT Eigenfunctions}

We have derived the operator $\mathcal{C}_{\mathbf{M}}$ in the form of the second-order differential equation. However, we do not know the explicit form of the eigenvalues $\mu_{\mathbf{M}}$ and the connection to $\lambda_{\mathbf{M}}$.

In the following discussion, we follow the same category as that in [14]. However, the case of $|a+d|>2$ and $b=0$ is discussed separately, because the existing results (Case F and Case $\mathrm{G}$ in Table 1 ) have undefined integration kernels. This further divides Case (F) and Case (G) into two sub-cases (F1)/(F2) and two sub-cases (G1)/(G2). The definitions of these cases are listed in Fig. 1. In each case, the closed-form eigenfunctions are derived and Property 2 and Property 4 are examined to consolidate the properties of the derived eigenfunctions. 


\section{A. Case (A): $|a+d|<2$}

In case (A), the eigenfunctions and the eigenvalues are already known in [4] and they are summarized in Case (A) of Table 1. In addition, the differential equations for the eigenfunctions were also derived in [4], which are

$$
\begin{aligned}
& \left(-\frac{1}{2} \sigma^{2} \mathcal{D}_{t}^{2}-j \tau t \mathcal{D}_{t}+\frac{1+\tau^{2}}{2 \sigma^{2}} t^{2}-\frac{j \tau}{2}\right) \psi_{n}(t) \\
& =(n+1 / 2) \psi_{n}(t) .
\end{aligned}
$$

Here we denote the eigenfunctions as $\psi_{n}(t)$ because they depend on the parameter $n=$ $0,1,2 \ldots$ Starting from (24), substituting parameter $\lambda, \sigma$, and $\tau$ into $a, b, c, d$, as summarized in Case (A) of Table 1, and multiplying $-(2 b) / \sigma^{2}$ on both sides give us

$$
\begin{aligned}
& \left(b \mathcal{D}_{t}^{2}+j(a-d) t \mathcal{D}_{t}+c t^{2}-j \frac{a-d}{2}\right) \psi_{n}(t) \\
& =-\left(2 b / \sigma^{2}\right)(n+1 / 2) \psi_{n}(t),
\end{aligned}
$$

Also, because of the multiplication property of the differential operator, $\mathcal{D}_{t} t=t \mathcal{D}_{t}+\mathcal{I}$, where $\mathcal{I} x(t)=x(t)$ is the identity operator, we arrange the above equation into

$$
\begin{aligned}
\mathcal{C}_{\mathbf{M}} \psi_{n}(t) & =\left(b \mathcal{D}_{t}^{2}+j \frac{a-d}{2}\left(t \mathcal{D}_{t}+\mathcal{D}_{t} t\right)+c t^{2}\right) \psi_{n}(t) \\
& =-\operatorname{sgn}(b) \sqrt{4-(a+d)^{2}}(n+1 / 2) \psi_{n}(t) .
\end{aligned}
$$

Hence, the existing differential equations are matched with our general operator $\mathcal{C}_{\mathbf{M}}$. The eigenvalues $\mu_{\mathrm{M}}$ are found to be

$$
\mu_{\mathbf{M}}=-\operatorname{sgn}(b) \sqrt{4-(a+d)^{2}}(n+1 / 2) .
$$

The next problem is to find the closed-form expression of $\lambda_{\mathbf{M}}$ in terms of $\mu_{\mathbf{M}}$. Still, from the existing result in Table 1 , we obtain

$$
\lambda_{\mathbf{M}}=e^{-j(n+1 / 2) \alpha}=(\cos \alpha+j \sin \alpha)^{-(n+1 / 2)} .
$$

(28) is associated with parameters $\alpha$ and $n$. Substituting $\sin \alpha, \cos \alpha$ into the form given in Table 1, which are

$$
\begin{aligned}
& \cos \alpha=(a+d) / 2, \\
& \sin \alpha=\frac{1}{2} \operatorname{sgn}(b) \sqrt{4-(a+d)^{2}},
\end{aligned}
$$

and $n+1 / 2$ into $\mu_{\mathbf{M}}$ by $(27), \lambda_{\mathbf{M}}$ can be written as

$$
\lambda_{\mathrm{M}}=\left[\frac{a+d}{2}+j \frac{\operatorname{sgn}(b) \sqrt{4-(a+d)^{2}}}{2}\right]^{\frac{\mu_{\mathrm{M}}}{\operatorname{sgn}(b) \sqrt{4-(a+d)^{2}}}} .
$$


The reason why $\lambda_{\mathbf{M}}$ is written as (31) is that we want to find the direct relationship among $a, b, c, d, \mu_{\mathbf{M}}$ and $\lambda_{\mathbf{M}}$ instead of defining other intermediate parameters such as $\sigma, \alpha, \tau$. In other cases, we take this direct approach to derive the formula concerning $\mu_{\mathbf{M}}$ and $\lambda_{\mathbf{M}}$.

We briefly examine these results. The eigenfunctions have been known to be complete and orthogonal [4]. According to (28), the eigenvalues $\left|\lambda_{\mathbf{M}}\right|=1$,

$$
\left|\lambda_{\mathbf{M}}\right|=|\cos \alpha+j \sin \alpha|^{-(n+1 / 2)}=1 \text {. }
$$

4.B. Case (B): $a+d=2, b=0$

In this case, $a+d=2$ and $b=0$. The matrix is $\mathbf{M}=[1,0 ; c, 1]$. This operation is the chirp-multiplication. In [14], the solution to this case is known as the linear combination of the delta functions. We want to see whether our operator $\mathcal{C}_{\mathrm{M}}$ gives us the same result. Then, the complete and orthogonal eigenfunctions are to be found.

Starting from $\mathcal{C}_{\mathbf{M}}$, the differential equation in this case becomes

$$
c t^{2} \psi_{\mu_{\mathbf{M}}}(t)=\mu_{\mathbf{M}} \psi_{\mu_{\mathbf{M}}}(t) .
$$

Dividing both sides by $c$ gives us

$$
t^{2} \psi_{\mu_{\mathbf{M}}}(t)=\frac{\mu_{\mathbf{M}}}{c} \psi_{\mu_{\mathbf{M}}}(t)
$$

Because the variable $t \in \mathbb{R}, t^{2}$ is non-negative and $\mu_{\mathbf{M}} / c$ is also non-negative. Note that $\mu_{\mathbf{M}}$ is no longer limited to non-negative integers. We are free to choose $\mu_{\mathbf{M}}$ as long as $\mu_{\mathbf{M}} / c$ is a non-negative real number. The two possible solutions to (34) are the delta functions

$$
\begin{aligned}
& \psi_{\mu_{\mathbf{M}}}^{(1, B)}(t)=\delta\left(t+\sqrt{\mu_{\mathbf{M}} / c}\right), \\
& \psi_{\mu_{\mathbf{M}}}^{(2, B)}(t)=\delta\left(t-\sqrt{\mu_{\mathbf{M}} / c}\right),
\end{aligned}
$$

where the position of the delta functions is either $-\sqrt{\mu_{\mathbf{M}} / c}$ in the first solution or $\sqrt{\mu_{\mathbf{M}} / c}$ in the second solution. The notation " $(1, B)$ " in the superscript stands for the first solution to Case (B).

Taking the LCT on both sides of (35) and (36) gives us

$$
\begin{aligned}
& \mathcal{L}_{\mathbf{M}} \psi_{\mu_{\mathbf{M}}}^{(1, B)}(t)=e^{j \frac{1}{2} \mu_{\mathbf{M}}} \psi_{\mu_{\mathbf{M}}}^{(1, B)}(t), \\
& \mathcal{L}_{\mathbf{M}} \psi_{\mu_{\mathbf{M}}}^{(2, B)}(t)=e^{j \frac{1}{2} \mu_{\mathbf{M}}} \psi_{\mu_{\mathbf{M}}}^{(2, B)}(t) .
\end{aligned}
$$

Then the eigenvalues $\lambda_{\mathbf{M}}=e^{j \frac{1}{2} \mu_{\mathbf{M}}}$. We can briefly check the properties of the derived eigenvalues and the eigenfunctions. First of all, the eigenvalues $\mu_{\mathbf{M}}$ are surely real numbers and it is trivial that $\left|\lambda_{\mathbf{M}}\right|=1$. For the eigenvalues, as long as two delta functions locate on different positions, they are always orthogonal, i.e.

$$
\left\langle\psi_{\mu_{\mathbf{M}}}^{(1, B)}(t), \psi_{\mu_{\mathbf{M}}^{\prime}}^{(1, B)}(t)\right\rangle=\delta\left(\mu_{\mathbf{M}}-\mu_{\mathbf{M}}^{\prime}\right)
$$


where $\langle x(t), y(t)\rangle=\int_{-\infty}^{\infty} x^{*}(t) y(t) \mathrm{d} t$ denotes the inner product of two functions. On the other hand, for the second solution $\psi_{\mu_{\mathbf{M}}}^{(2, B)}(t)$, we have the following orthogonality relationship:

$$
\begin{aligned}
& \left\langle\psi_{\mu_{\mathbf{M}}}^{(1, B)}(t), \psi_{\mu_{\mathbf{M}}^{\prime}}^{(2, B)}(t)\right\rangle= \begin{cases}\delta\left(\mu_{\mathbf{M}}-\mu_{\mathbf{M}}^{\prime}\right) & \mu_{\mathbf{M}}=\mu_{\mathbf{M}}^{\prime}=0, \\
0 & \text { otherwise, }\end{cases} \\
& \left\langle\psi_{\mu_{\mathbf{M}}}^{(2, B)}(t), \psi_{\mu_{\mathbf{M}}^{\prime}}^{(2, B)}(t)\right\rangle=\delta\left(\mu_{\mathbf{M}}-\mu_{\mathbf{M}}^{\prime}\right) .
\end{aligned}
$$

As a result, these functions are really orthogonal to each other (see Appendix A for details).

Next, we want to relate our results to those in [14]. In Case (B) of Table 1, the eigenvalues are the same if $\mu_{\mathbf{M}}=c h$. However, the eigenfunctions are linear combination of different delta function located non-uniformly on the $t$-axis. The frequency spectra of those eigenfunctions result in almost-periodic functions [14]. If $\mu_{\mathbf{M}}=c h$, each term in the summation is exactly in the following form of $\psi_{\mu_{\mathbf{M}}}^{(1, B)}(t)$ or $\psi_{\mu_{\mathbf{M}}}^{(2, B)}(t)$. The eigenvalues $\lambda_{\mathbf{M}}$ and the derived eigenfunctions are related as follows

$$
\begin{aligned}
& {\left[\text { The eigenvalue of } \psi_{\mu_{\mathbf{M}}+4 n \pi}^{(1, B)}(t)\right]=e^{j \frac{1}{2}\left(\mu_{\mathbf{M}}+4 n \pi\right)}=e^{j \frac{1}{2} \mu_{\mathbf{M}}},} \\
& {\left[\text { The eigenvalue of } \psi_{\mu_{\mathbf{M}}+4 m \pi}^{(2, B)}(t)\right]=e^{j \frac{1}{2}\left(\mu_{\mathbf{M}}+4 m \pi\right)}=e^{j \frac{1}{2} \mu_{\mathbf{M}}},} \\
& {\left[\text { The eigenvalue of } \sum_{n} A_{n} \psi_{\mu_{\mathbf{M}}+4 n \pi}^{(1, B)}(t)+B_{n} \psi_{\mu_{\mathbf{M}}+4 n \pi}^{(2, B)}(t)\right]=e^{j \frac{1}{2} \mu_{\mathbf{M}}} .}
\end{aligned}
$$

Based on the above discussion, our results, (44), are exactly equivalent to the existing results. However, the existing results are not always orthogonal, depending on the combination coefficients $A_{n}$ and $B_{n}$. Our solution features the simplicity and the orthogonality of the eigenfunctions.

\section{C. Case (C): $a+d=-2, b=0$}

In this case, the matrix $\mathbf{M}$ is $[-1,0 ; c,-1]$. Therefore, the differential equation in this case is identical to (34) so there are still two solutions (35) and (36). However, if we take the LCT on the eigenfunctions, we obtain

$$
\begin{aligned}
& \mathcal{L}_{\mathbf{M}} \psi_{\mu_{\mathbf{M}}}^{(1, B)}(t)=j e^{-j \frac{1}{2} \mu_{\mathbf{M}}} \psi_{\mu_{\mathbf{M}}}^{(2, B)}(t), \\
& \mathcal{L}_{\mathbf{M}} \psi_{\mu_{\mathbf{M}}}^{(2, B)}(t)=j e^{-j \frac{1}{2} \mu_{\mathbf{M}}} \psi_{\mu_{\mathbf{M}}}^{(1, B)}(t) .
\end{aligned}
$$

The LCT of $\psi_{\mu_{\mathrm{M}}}^{(1, B)}(t)$ yields $\psi_{\mu_{\mathrm{M}}}^{(2, B)}(t)$ and vice versa. Combining (45) and (46) together gives us two equations

$$
\begin{aligned}
\mathcal{L}_{\mathbf{M}} \psi_{\mu_{\mathbf{M}}}^{(1, C)}(t) & =j e^{-j \frac{1}{2} \mu_{\mathbf{M}}} \psi_{\mu_{\mathbf{M}}}^{(1, C)}(t), \\
\mathcal{L}_{\mathbf{M}} \psi_{\mu_{\mathbf{M}}}^{(2, C)}(t) & =-j e^{-j \frac{1}{2} \mu_{\mathbf{M}}} \psi_{\mu_{\mathbf{M}}}^{(2, C)}(t),
\end{aligned}
$$


where

$$
\begin{aligned}
& \psi_{\mu_{\mathrm{M}}}^{(1, C)}(t)=\frac{1}{\sqrt{2}}\left(\psi_{\mu_{\mathrm{M}}}^{(1, B)}(t)+\psi_{\mu_{\mathrm{M}}}^{(2, B)}(t)\right) \\
& \psi_{\mu_{\mathrm{M}}}^{(2, C)}(t)=\frac{1}{\sqrt{2}}\left(\psi_{\mu_{\mathrm{M}}}^{(1, B)}(t)-\psi_{\mu_{\mathrm{M}}}^{(2, B)}(t)\right)
\end{aligned}
$$

According to $(47)$ and $(48), \psi_{\mu_{\mathbf{M}}}^{(1, C)}(t)$ and $\psi_{\mu_{\mathbf{M}}}^{(2, C)}(t)$ are all eigenfunctions. Besides, we notice that the eigenvalues are $\lambda_{\mathbf{M}}^{(1, C)}=j e^{-j \frac{1}{2} \mu_{\mathbf{M}}}$ for the first solution and $\lambda_{\mathbf{M}}^{(2, C)}=-j e^{-j \frac{1}{2} \mu_{\mathbf{M}}}$ for the second solution. It is trivial to show that $\mu_{\mathbf{M}}$ are real, $\left|\lambda_{\mathbf{M}}^{(1, C)}\right|=\left|\lambda_{\mathbf{M}}^{(2, C)}\right|=1$, and $\psi_{\mu_{\mathbf{M}}}^{(1, C)}(t)$ and $\psi_{\mu_{\mathbf{M}}}^{(2, C)}(t)$ are orthogonal to each other.

Our results are also equivalent to the previous results. In Case (B) of Table 1, the combination coefficients $A_{n}= \pm B_{n}$ must be satisfied. If the two terms involving $A_{n}$ and $B_{n}$ are combined, we have the form similar to $\psi_{\mu_{\mathbf{M}}}^{(1, C)}(t)$ or $\psi_{\mu_{\mathbf{M}}}^{(2, C)}(t)$ as long as $\mu_{\mathbf{M}}=c h$. Again, the existing solution is the general form of the eigenfunctions and our results are the orthonormal eigenfunctions.

However, we find that the previous results, (64) in [14], for the eigenvalues has a minor error in sign. The eigenvalues should be corrected as $\pm j e^{-j c h / 2}$. The calculation details are provided in Appendix B.

4.D. Case (D): $a+d=2, b \neq 0$

In this case, we follow the method proposed in [14]. The matrix is then decomposed as

$$
\left[\begin{array}{ll}
a & b \\
c & d
\end{array}\right]=\left[\begin{array}{ll}
a_{1} & b_{1} \\
c_{1} & d_{1}
\end{array}\right]\left[\begin{array}{ll}
1 & \eta \\
0 & 1
\end{array}\right]\left[\begin{array}{ll}
a_{1} & b_{1} \\
c_{1} & d_{1}
\end{array}\right]^{-1}=\mathbf{M}_{1} \mathbf{M}_{0} \mathbf{M}_{1}^{-1}
$$

where

$$
\eta=\frac{b}{a_{1}^{2}}, \quad c_{1}=\frac{d-a}{2 b} a_{1}, \quad b_{1}=\frac{2 b\left(d_{1}-a_{1}^{-1}\right)}{d-a}, a_{1} \neq 0
$$

and $d_{1}$ is free to choose. We denote $\mathbf{M}_{0}=[1, \eta ; 0,1]$ and $\mathbf{M}_{1}=\left[a_{1}, b_{1} ; c_{1}, d_{1}\right]$ for further discussion. The eigenfunctions for the $\mathbf{M}_{0}$ part can be simply derived. The operator $\mathcal{C}_{\mathbf{M}_{0}}$ is reduced to $\eta \mathcal{D}_{t}^{2}$ in this case. The eigenfunctions for $\mathbf{M}_{0}$, say $\psi_{\mu_{\mathbf{M}_{0}}}(t)$, satisfy

$$
\mathcal{D}_{t}^{2} \psi_{\mu_{\mathbf{M}_{0}}}(t)=\frac{\mu_{\mathbf{M}_{0}}}{\eta} \psi_{\mu_{\mathbf{M}_{0}}}(t)
$$

Because the operator on the left-hand side is self-adjoint, the eigenvalues $\mu_{\mathbf{M}_{0}} / \eta$ is real. If $\mu_{\mathbf{M}_{0}} / \eta$ is positive, the solution is $e^{ \pm t \sqrt{\mu_{\mathrm{M}_{0}} / \eta}}$, which is divergent due to positive exponent. It is inappropriate for real exponentials to be eigenfunctions. First of all, the orthogonal property (Property 2) is not satisfied. To verify it, if we take the inner product between $e^{t \sqrt{\mu_{\mathrm{M}_{0}} / \eta}}$ and $e^{t \sqrt{\mu_{\mathbf{M}_{0}}^{\prime} / \eta}}$ for $\mu_{\mathbf{M}_{0}} \neq \mu_{\mathbf{M}_{0}}^{\prime}$, the result becomes $\int_{-\infty}^{\infty} e^{t\left(\sqrt{\mu_{\mathbf{M}_{0}} / \eta}+\sqrt{\left.\mu_{\mathbf{M}_{0}}^{\prime} / \eta\right)}\right.} \mathrm{d} t$, which is divergent. 
Then, if we choose $\mu_{\mathbf{M}_{0}}=\mu_{\mathbf{M}_{0}}^{\prime}$ and compute the $l_{2}$-norm of the real exponentials, we have that $\left\|e^{t \sqrt{\mu_{\mathrm{M}_{0}} / \eta}}\right\|_{2}$ is also divergent so it is impossible to find the normalization factor, not to mention digital computation.

Hence, the solution for non-positive $\mu_{\mathbf{M}_{0}} / \eta$ is the complex exponential function, which is explicitly

$$
\begin{aligned}
& \psi_{\mu_{\mathbf{M}_{0}}}^{(1, D)}(t)=\frac{1}{\sqrt{2 \pi}} e^{j \sqrt{\frac{-\mu_{\mathbf{M}_{0}}}{\eta} t},} \\
& \psi_{\mu_{\mathbf{M}_{0}}}^{(2, D)}(t)=\frac{1}{\sqrt{2 \pi}} e^{-j \sqrt{\frac{-\mu_{\mathbf{M}_{0}}}{\eta} t}} .
\end{aligned}
$$

The solution is dual to the solution in Case (B), (35) and (36). The eigenvalues for these two solutions are $\lambda_{\mathbf{M}_{0}}=e^{j \frac{1}{2} \mu_{\mathbf{M}_{0}}}$.

Then we want to derive the corresponding differential equation for the actual eigenfunction $\psi_{\mu_{\mathbf{M}}}(t)$. From (53), taking LCT of $\mathbf{M}_{1}$ on both sides yields

$$
\mathcal{L}_{\mathbf{M}_{1}} \mathcal{D}_{t}^{2} \psi_{\mu_{\mathbf{M}_{0}}}(t)=\frac{\mu_{\mathbf{M}_{0}}}{\eta} \mathcal{L}_{\mathbf{M}_{1}} \psi_{\mu_{\mathbf{M}_{0}}}(t)
$$

Applying (11) to (56) leads to

$$
\eta\left(a_{1} \mathcal{D}_{t}-j c_{1} t\right)^{2} \mathcal{L}_{\mathbf{M}_{1}} \psi_{\mu_{\mathbf{M}_{0}}}(t)=\mu_{\mathbf{M}_{0}} \mathcal{L}_{\mathbf{M}_{1}} \psi_{\mu_{\mathbf{M}_{0}}}(t)
$$

Then we expand the operator on the left-hand side and utilize Property B in [14], which is $\psi_{\mu_{\mathrm{M}}}(t)=\mathcal{L}_{\mathbf{M}_{1}} \psi_{\mu_{\mathrm{M}_{0}}}(t)$,

$$
\begin{aligned}
& {\left[a_{1}^{2} \eta \mathcal{D}_{t}^{2}-j a_{1} c_{1} \eta\left(t \mathcal{D}_{t}+\mathcal{D}_{t} t\right)-c_{1}^{2} \eta t^{2}\right] \psi_{\mu_{\mathbf{M}}}(t) } \\
= & \mu_{\mathbf{M}_{0}} \psi_{\mu_{\mathbf{M}}}(t)
\end{aligned}
$$

According to (52), the coefficients on the left-hand side operator in (58) are

$$
a_{1}^{2} \eta=b, \quad-j a_{1} c_{1} \eta=j \frac{a-d}{2},-c_{1}^{2} \eta=c .
$$

Then we notice that (58) is exactly

$$
\mathcal{C}_{\mathbf{M}} \psi_{\mu_{\mathbf{M}}}(t)=\mu_{\mathbf{M}} \psi_{\mu_{\mathbf{M}}}(t)
$$

where $\mu_{\mathbf{M}}=\mu_{\mathbf{M}_{0}}$ It is proved in (60) that the eigenfunctions are associated with the operator $\mathcal{C}_{\mathrm{M}}$.

Since we find the closed-form expression, $\phi_{\mu_{\mathbf{M}_{0}}}^{(1, D)}(t)$ and $\phi_{\mu_{\mathbf{M}_{0}}}^{(2, D)}(t)$, we can continue the derivation in [14]. We start from the integral expression, listed in Case (D) of Table 1 , set $a_{1}=1$, $d_{1}=0$ and use the Gaussian integrals

$$
\int_{-\infty}^{\infty} e^{-\alpha \xi^{2}+\beta \xi} \mathrm{d} \xi=\sqrt{\frac{\pi}{\alpha}} e^{\frac{\beta^{2}}{4 \alpha}},
$$


to have the following results

$$
\begin{aligned}
\psi_{\mu_{\mathrm{M}}}^{(1, D)}(t) & =\frac{1}{\sqrt{2 \pi}} e^{j \frac{d-a}{4 b} t^{2}} e^{j t \sqrt{-\mu_{\mathrm{M}} / b}}, \\
\psi_{\mu_{\mathrm{M}}}^{(2, D)}(t) & =\frac{1}{\sqrt{2 \pi}} e^{j \frac{d-a}{4 b} t^{2}} e^{-j t \sqrt{-\mu_{\mathrm{M}} / b}} .
\end{aligned}
$$

In the derivation, the constant phase factor is dropped for simplicity. (62) and (63) are the final eigenfunctions for Case (D). The eigenvalues $\lambda_{\mathbf{M}}=\lambda_{\mathbf{M}_{0}}=e^{j \frac{1}{2} \mu_{\mathbf{M}}}$.

Here we briefly examine the results. The eigenvalues $\mu_{\mathbf{M}}$ are surely real, $\left|\lambda_{\mathbf{M}}\right|=1$, and the eigenfunctions are orthogonal because

$$
\begin{aligned}
& \left\langle\psi_{\mu_{\mathbf{M}}}^{(1, D)}(t), \psi_{\mu_{\mathbf{M}}^{\prime}}^{(1, D)}(t)\right\rangle \\
= & \left\langle\psi_{\mu_{\mathbf{M}_{0}}}^{(1, D)}(t), \psi_{\mu_{\mathbf{M}_{0}}^{\prime}}^{(1, D)}(t)\right\rangle=\delta\left(\mu_{\mathbf{M}_{0}}-\mu_{\mathbf{M}_{0}}^{\prime}\right),
\end{aligned}
$$

so does the second solution $\psi_{\mu_{\mathrm{M}}}^{(2, D)}(t)$ (see Appendix A for the inner product of complex exponential functions). Besides, when $a=d=1,(62)$ is reduced to (54) and (63) is reduced to $(55)$.

Finally, we relate our results to the previous results. In Case (D) of Table 1, the function $g(t)$ serves as the same role of $\psi_{\mu_{\mathbf{M}_{0}}}^{(1, D)}(t)$ and $\psi_{\mu_{\mathbf{M}_{0}}}^{(2, D)}(t) . g(t)$ are just linear combinations of them. The parameter $\mu_{\mathbf{M}}$ is identical to $-b h$ in [14]. In addition, we utilize the Gaussian integral to write out a closed-form expression of the eigenfunctions, which is more elegant than the previous form of eigenfunctions.

4.E. Case (E): $a+d=-2, b \neq 0$

Following the derivation in Case (D), we have

$$
\left[\begin{array}{ll}
a & b \\
c & d
\end{array}\right]=\left[\begin{array}{ll}
a_{1} & b_{1} \\
c_{1} & d_{1}
\end{array}\right]\left[\begin{array}{cc}
-1 & \eta \\
0 & -1
\end{array}\right]\left[\begin{array}{ll}
a_{1} & b_{1} \\
c_{1} & d_{1}
\end{array}\right]^{-1},
$$

The parameters share the same relationship as (52). We also write $\mathbf{M}_{0}=[-1, \eta ; 0,-1]$ and $\mathbf{M}_{1}=\left[a_{1}, b_{1} ; c_{1}, d_{1}\right]$. Following the derivation in Case (D), the eigenfunctions for $\mathbf{M}_{0}$ are found to be

$$
\begin{aligned}
\psi_{\mu_{\mathbf{M}_{0}}}^{(1, E)}(t) & =\frac{1}{\sqrt{2}}\left(\psi_{\mu_{\mathbf{M}_{0}}}^{(1, D)}(t)+\psi_{\mu_{\mathbf{M}_{0}}}^{(2, D)}(t)\right) \\
& =\frac{1}{\sqrt{\pi}} \cos \left(\sqrt{\frac{-\mu_{\mathbf{M}_{0}}}{\eta}} t\right), \\
\psi_{\mu_{\mathbf{M}_{0}}}^{(2, E)}(t) & =\frac{1}{\sqrt{2}}\left(\psi_{\mu_{\mathbf{M}_{0}}}^{(1, D)}(t)-\psi_{\mu_{\mathbf{M}_{0}}}^{(2, D)}(t)\right) \\
& =\frac{j}{\sqrt{\pi}} \sin \left(\sqrt{\frac{-\mu_{\mathbf{M}_{0}}}{\eta}} t\right) .
\end{aligned}
$$


The eigenvalues for $\mathbf{M}_{0}$ are $\lambda_{\mathbf{M}_{0}}=e^{-\frac{j}{2}\left(\mu_{\mathbf{M}_{0}} \pm \operatorname{sgn}(b) \pi\right)}$ for the first/second solution.

To find the actual eigenfunctions, we take LCT for $\mathbf{M}_{1}$ on both sides of (66) and (68) and using the results in Case (D), (62) and (63), the actual eigenfunctions are

$$
\begin{aligned}
& \psi_{\mu_{\mathbf{M}}}^{(1, E)}(t)=\frac{1}{\sqrt{\pi}} e^{j \frac{d-a}{4 b} t^{2}} \cos \left(\sqrt{\frac{-\mu_{\mathbf{M}}}{b}} t\right), \\
& \psi_{\mu_{\mathbf{M}}}^{(2, E)}(t)=\frac{j}{\sqrt{\pi}} e^{j \frac{d-a}{4 b} t^{2}} \sin \left(\sqrt{\frac{-\mu_{\mathbf{M}}}{b}} t\right),
\end{aligned}
$$

where the constant phase factor $j$ in $\psi_{\mu_{\mathrm{M}}}^{(2, E)}$ can be neglected to make the solutions more symmetrical. Still, the eigenvalues are then $\lambda_{\mathbf{M}}=e^{-\frac{j}{2}\left(\mu_{\mathbf{M}} \pm \operatorname{sgn}(b) \pi\right)}$ for the first/second solution. In addition, it is trivial to show that $\psi_{\mu_{\mathbf{M}}}^{(1, E)}(t)$ and $\psi_{\mu_{\mathbf{M}}}^{(2, E)}(t)$ are the eigenfunctions of $\mathcal{C}_{\mathbf{M}}$ with eigenvalues $\mu_{\mathrm{M}}$.

It is not difficult to verify that $\mu_{\mathbf{M}}$ is real, $\left|\lambda_{\mathbf{M}}\right|=1$, and the eigenfunctions are orthogonal, based on the previous results. The results are also equivalent to those in [14]. The even/odd combination of Case (D) gives us the eigenfunctions of Case (E). The eigenvalues are a little different due to the details described in Case (C).

\section{F. $\operatorname{Case}(F): a+d>2$}

Here we want to discuss the most complicated eigenfunctions for $a+d>2$. The eigenfunctions in the case were investigated in [14]. The matrix was decomposed into

$$
\left[\begin{array}{ll}
a & b \\
c & d
\end{array}\right]=\left[\begin{array}{ll}
a_{1} & b_{1} \\
c_{1} & d_{1}
\end{array}\right]\left[\begin{array}{cc}
\sigma^{-1} & 0 \\
0 & \sigma
\end{array}\right]\left[\begin{array}{ll}
a_{1} & b_{1} \\
c_{1} & d_{1}
\end{array}\right]^{-1},
$$

where

$$
\begin{aligned}
\sigma & =\frac{a+d \pm \sqrt{(a+d)^{2}-4}}{2}>0 \\
s & =\operatorname{sgn}\left(\sigma-\sigma^{-1}\right) \\
b_{1} & =\frac{s b}{a_{1} \sqrt{(a+d)^{2}-4}}, \\
c_{1} & =\frac{-2 a_{1} s c}{s(d-a)+\sqrt{(a+d)^{2}-4}} \\
d_{1} & =\frac{1}{2 a_{1}}\left(\frac{s(d-a)}{\sqrt{(a+d)^{2}-4}}+1\right)
\end{aligned}
$$

and $a_{1}$ is free to choose, except 0 . We further define $\mathbf{M}_{0}=\left[\sigma^{-1}, 0 ; 0, \sigma\right]$ and $\mathbf{M}_{1}=$ $\left[a_{1}, b_{1} ; c_{1}, d_{1}\right]$ for simplicity. It was also claimed in [14] that the eigenfunctions are

$$
\psi_{\mu_{\mathbf{M}}}(t)=\mathcal{L}_{\mathbf{M}_{1}} h(t)
$$


where

$$
\sqrt{\sigma} h(\sigma t)=\lambda h(t)
$$

Besides, $\lambda$ are exactly the eigenvalues for the LCT. However, in [14], there is no closed-form solution for $h(t)$ and $\lambda$.

The solution to (79) seems quite complicated. However, this equation has been known widely as the transform kernel of the Mellin transform [25], which can be reduced to the scale transform in some cases [16,26-29]. In [26], the eigenfunctions of the scaling operation were found to be

$$
h_{\omega}(t)= \begin{cases}\frac{1}{\sqrt{2 \pi}} t^{-\frac{1}{2}+j \omega} & t \in(0, \infty), \\ 0 & t \in(-\infty, 0],\end{cases}
$$

where $\omega \in \mathbb{R}$. Here we modify the notation a little to be consistent with the notation in this paper. We can prove that (80) is the solution to (79). For instance, when $t>0$, we have

$$
\begin{aligned}
\sqrt{\sigma} h_{\omega}(\sigma t) & =\sqrt{\sigma} \frac{(\sigma t)^{-\frac{1}{2}+j \omega}}{\sqrt{2 \pi}}=\sqrt{\sigma} \sigma^{-\frac{1}{2}+j \omega} \frac{t^{-\frac{1}{2}+j \omega}}{\sqrt{2 \pi}} \\
& =\sigma^{j \omega} \frac{t^{-\frac{1}{2}+j \omega}}{\sqrt{2 \pi}}=e^{j \omega \log \sigma} h_{\omega}(t) .
\end{aligned}
$$

Hence, (80) are really the eigenfunctions of the scaling operation. It was also discussed in [26] that $h_{\omega}(t)$ are complete and orthonormal and $h_{\omega}(t)$ obey the differential equation

$$
\frac{1}{2 j}\left(t \mathcal{D}_{t}+\mathcal{D}_{t} t\right) h_{\omega}(t)=\omega h_{\omega}(t)
$$

In [26], $h_{\omega}(t)$ is the transform kernel of the scale transform.

In addition to $h_{\omega}(t), h_{\omega}(-t)$ is also the solution to (79) and (82). To be consistent with the notation in this paper, we write $h_{\omega}(t)$ and $h_{\omega}(-t)$ to be

$$
\begin{aligned}
& \psi_{\mu_{\mathrm{M}_{0}}}^{(1, F 1)}(t)=h_{\omega}(t)= \begin{cases}\frac{1}{\sqrt{2 \pi}} t^{-\frac{1}{2}+j \omega} & t \in(0, \infty), \\
0 & t \in(-\infty, 0],\end{cases} \\
& \psi_{\mu_{\mathrm{M}_{0}}}^{(2, F 1)}(t)=h_{\omega}(-t)= \begin{cases}\frac{1}{\sqrt{2 \pi}}(-t)^{-\frac{1}{2}+j \omega} & t \in(-\infty, 0), \\
0 & t \in[0, \infty) .\end{cases}
\end{aligned}
$$

It is for sure that the linear combinations of $\psi_{\mu_{\mathbf{M}_{0}}}^{(1, F 1)}(t)$ and $\psi_{\mu_{\mathbf{M}_{0}}}^{(2, F)}(t)$ are all the eigenfunctions of the scaling operation. Based on our notation, the eigenfunctions are specified by $\mu_{\mathbf{M}}$; however, the existing solution to (82) is expressed in $\omega$. The connection between $\mu_{\mathbf{M}}$ and $\omega$ will be derived later.

With the closed-form solution for eigenfunctions of the scaling operation, we can continue the rest work for the eigenfunctions in this case. First of all, we want to derive the differential 
equation for the actual eigenfunctions $\psi_{\mu_{\mathbf{M}}}(t)$. Starting from $(82)$ and taking $\mathcal{L}_{\mathbf{M}_{1}}$ on both sides, we obtain

$$
\frac{1}{2 j} \mathcal{L}_{\mathbf{M}_{1}}\left[\left(t \mathcal{D}_{t}+\mathcal{D}_{t} t\right) \psi_{\mu_{\mathrm{M}_{0}}}(t)\right]=\omega \mathcal{L}_{\mathbf{M}_{1}} \psi_{\mu_{\mathrm{M}_{0}}}(t)
$$

Using the property of the differential operator $\mathcal{D}_{t} t=t \mathcal{D}_{t}+\mathcal{I}$ and the relationship between the actual eigenfunctions and the scaling eigenfunctions $\psi_{\mu_{\mathrm{M}}}(t)=\mathcal{L}_{\mathbf{M}_{1}} \psi_{\mu_{\mathrm{M}_{0}}}(t)$, we have

$$
\frac{1}{2 j} \mathcal{L}_{\mathbf{M}_{1}}\left[\left(2 t \mathcal{D}_{t}+\mathcal{I}\right) \psi_{\mu_{\mathbf{M}_{0}}}(t)\right]=\omega \psi_{\mu_{\mathbf{M}}}(t)
$$

According (6) and (7), replacing the operators yields

$$
\begin{aligned}
& \frac{1}{2 j}\left[2\left(j b_{1} \mathcal{D}_{t}+t d_{1}\right)\left(a_{1} \mathcal{D}_{t}-j c_{1} t\right)+1\right] \psi_{\mu_{M}}(t) \\
= & \omega \psi_{\mu_{M}}(t) .
\end{aligned}
$$

Rearranging these terms gives us

$$
\begin{aligned}
& {\left[a_{1} b_{1} \mathcal{D}_{t}^{2}+\frac{2 a_{1} d_{1}-1}{2 j}\left(t \mathcal{D}_{t}+\mathcal{D}_{t} t\right)-c_{1} d_{1} t^{2}\right] \psi_{\mu_{\mathrm{M}}}(t) } \\
= & \omega \psi_{\mu_{\mathrm{M}}}(t) .
\end{aligned}
$$

Then, from (73) to (77), we can modify the differential equation into

$$
\mathcal{C}_{\mathbf{M}} \psi_{\mu_{\mathbf{M}}}(t)=\operatorname{sgn}\left(\sigma-\sigma^{-1}\right) \omega \sqrt{(a+d)^{2}-4} \psi_{\mu_{\mathbf{M}}}(t)
$$

According to (89), the eigenfunction is also the solution to the operator $\mathcal{C}_{\mathbf{M}}$. The eigenvalues are then

$$
\mu_{\mathbf{M}}=\operatorname{sgn}\left(\sigma-\sigma^{-1}\right) \omega \sqrt{(a+d)^{2}-4} .
$$

The next question is the eigenvalue for LCT $\lambda_{\mathbf{M}}$. From [14], the eigenvalue is found in the solution of (79), which is

$$
\begin{aligned}
\lambda_{\mathbf{M}} & =\frac{\sqrt{\sigma} \psi_{\mu_{\mathbf{M}_{0}}}(\sigma t)}{\psi_{\mu_{\mathbf{M}_{0}}}(t)}=\frac{\sqrt{\sigma}(\sigma t)^{-\frac{1}{2}+j \omega}}{t^{-\frac{1}{2}+j \omega}}=\sigma^{j \omega} \\
& =e^{j \omega \log \sigma}=e^{j \frac{\mu_{\mathbf{M}}}{\operatorname{sgn}\left(\sigma-\sigma^{-1}\right) \sqrt{(a+d)^{2}-4}} \log \sigma} .
\end{aligned}
$$

Hence, we relate the eigenvalue $\mu_{\mathbf{M}}$ with the eigenvalue for LCT, $\lambda_{\mathbf{M}}$.

4.F.1. $\quad$ Case (F1): $a+d>2, b \neq 0$

The final problem is the closed-form solution for the eigenfunctions. The integral form for the eigenfunction has been proposed in [14]. The result is very complicated and expressed in 
integrals. Here we choose $\psi_{\mu_{\mathbf{M}_{0}}}^{(1, F 1)}(t)$ for discussion. Taking $\mathcal{L}_{\mathbf{M}_{1}}$ on both sides of (83) gives us

$$
\begin{aligned}
& \psi_{\mu_{\mathbf{M}}}^{(1, F 1)}(t)=\mathcal{L}_{\mathbf{M}_{1}} \psi_{\mu_{\mathbf{M}_{0}}}^{(1, F 1)}(t) \\
= & \sqrt{\frac{1}{j 2 \pi b_{1}}} \int_{0}^{\infty}\left(e^{j \frac{a_{1}}{2 b_{1}} \xi^{2}} e^{-j \frac{t \xi}{b_{1}}} e^{j \frac{d_{1}}{2 b_{1}} t^{2}}\right) \frac{\xi^{-\frac{1}{2}+j \omega}}{\sqrt{2 \pi}} \mathrm{d} \xi
\end{aligned}
$$

for $b_{1} \neq 0$. If $b_{1}=0$, i.e. $b=0$, we have to seek another definition of LCT. We choose the condition of $a+d>2, b \neq 0$ for Case (F1) and the condition of $a+d>2, b=0$ for Case (F2). In (92), we can regard $\xi^{-\frac{1}{2}+j \omega} / \sqrt{2 \pi}$ as the transform kernel from $\xi$ to $\omega$ and $e^{j \frac{a_{1}}{2 b_{1}} \xi^{2}} e^{-j \frac{t \xi}{b_{1}}} e^{j \frac{d_{1}}{2 b_{1}} t^{2}}$ is regarded as the function in $\xi$ to be transformed. In [30], 3.13, we have the existing result for the integral of this form. It is

$$
\int_{0}^{\infty} e^{-\alpha \tau^{2}-\beta \tau} \tau^{\zeta-1} \mathrm{~d} \tau=(2 \alpha)^{-\frac{\zeta}{2}} \Gamma(\zeta) e^{\frac{\beta^{2}}{8 \alpha}} D_{-\zeta}\left(\frac{\beta}{\sqrt{2 \alpha}}\right)
$$

where $\Re \zeta>0$ and $\Re$ stands for the real part of a complex number. $\Gamma(z)$ is the gamma function defined by [31]

$$
\Gamma(z)=\int_{0}^{\infty} e^{-t} t^{z-1} \mathrm{~d} t
$$

$D_{\nu}(z)$ are the parabolic cylinder functions, which are the solution to the following differential equation

$$
\left(\mathcal{D}_{z}^{2}+\nu+\frac{1}{2}-\frac{1}{4} z^{2}\right) D_{\nu}(z)=0 .
$$

When $\nu$ is a non-negative integer, the parabolic cylinder function is reduced to the scaled Hermite Gaussian function [31]. In some references [31], the parabolic cylinder functions are denoted by $U(a, z)$, which are related to $D_{\nu}(z)$ by

$$
U(a, z)=D_{-a-\frac{1}{2}}(z)
$$

In (93), making the following change of variables

$$
\alpha=-j \frac{a_{1}}{2 b_{1}}, \quad \beta=\frac{j t}{b_{1}}, \quad \zeta=\frac{1}{2}+j \omega,
$$

gives us the following result

$$
\psi_{\mu_{\mathrm{M}}}^{(1, F 1)}(t)=N_{\omega} D_{-\frac{1}{2}-j \omega}\left(\frac{j e^{j \frac{\pi}{4}} t}{\sqrt{a_{1} b_{1}}}\right) e^{j \frac{2 a_{1} d_{1}-1}{4 a_{1} b_{1}} t^{2}},
$$

where $N_{\omega}$ is the factor given by

$$
N_{\omega}=\sqrt{\frac{1}{j 4 \pi^{2} b_{1}}}\left(\frac{-j a_{1}}{b_{1}}\right)^{-\frac{1}{4}-j \frac{\omega}{2}} \Gamma\left(\frac{1}{2}+j \omega\right) .
$$


Finally, substituting $a_{1}$ and $b_{1}$ into $a, b, c$, and $d$ yields our eigenfunctions of the LCT when $a+d>2, b \neq 0$.

$$
\psi_{\mu_{\mathrm{M}}}^{(1, F 1)}(t)=N_{\omega} D_{-\frac{1}{2}-j \omega}\left(\frac{e^{j \frac{3 \pi}{4}} t}{\sqrt{\eta}}\right) e^{j \frac{d-a}{4 b} t^{2}},
$$

where $\eta=s b / \sqrt{(a+d)^{2}-4}$, as defined in (127) in [14]. Here we have successfully obtained the closed-form eigenfunctions $\psi_{\mu_{\mathrm{M}}}^{(1, F 1)}(t)$ instead of integral expressions.

In (99), if we express $b_{1}$ in terms of $a_{1}$ by (75), it seems unreasonable that the normalization factor $N_{\omega}$ is still associated with $a_{1}$, which is free to choose. However, the modulus of $N_{\omega}$ is

$$
\left|N_{\omega}\right|=\frac{e^{-\frac{\pi \omega}{4}}}{\sqrt{2 \pi}} \eta^{-\frac{1}{4}}\left|\Gamma\left(\frac{1}{2}+j \omega\right)\right|,
$$

which is completely independent of $a_{1}$. Hence, varying $a_{1}$ only causes phase changes on the eigenfunctions and these multiple solutions are the same in essence. To remove the phase ambiguity, we use $\left|N_{\omega}\right|$ as the normalization factor instead of $N_{\omega}$.

For the eigenfunctions derived from the second solution, we follow the same procedure and have the second eigenfunctions

$$
\psi_{\mu_{\mathrm{M}}}^{(2, F 1)}(t)=\left|N_{\omega}\right| D_{-\frac{1}{2}-j \omega}\left(\frac{-e^{j \frac{3 \pi}{4}} t}{\sqrt{\eta}}\right) e^{j \frac{d-a}{4 b} t^{2}} .
$$

It is noted that $\psi_{\mu_{\mathrm{M}}}^{(2, F 1)}(t)=\psi_{\mu_{\mathrm{M}}}^{(1, F 1)}(-t)$, which are exactly the time-reversal of the first solution. The eigenvalues $\lambda_{\mathbf{M}}$ is identical to those in (91).

We verify the solutions of these eigenvalues and the eigenfunctions briefly. The eigenvalues $\mu_{\mathbf{M}}$ are all real due to (89). The eigenvalues $\lambda_{\mathbf{M}}$, as in (91) are located on the unit circle of the complex plane. The orthogonality of the eigenfunctions is also trivial. For instance, the first solutions $\psi_{\mu_{\mathbf{M}}}^{(1, F 1)}(t)$ are orthogonal to each other because

$$
\begin{aligned}
\left\langle\psi_{\mu_{\mathbf{M}}}^{(1, F 1)}(t), \psi_{\mu_{\mathbf{M}}^{\prime}}^{(1, F 1)}(t)\right\rangle & =\left\langle\psi_{\mu_{\mathbf{M}_{0}}}^{(1, F 1)}(t), \psi_{\mu_{\mathbf{M}_{0}}^{\prime}}^{(1, F 1)}(t)\right\rangle \\
& =\delta\left(\mu_{\mathbf{M}_{0}}-\mu_{\mathbf{M}_{0}}^{\prime}\right)
\end{aligned}
$$

The last equality is based on the fact that $\psi_{\mu_{\mathbf{M}_{0}}}^{(1, F 1)}(t)$ are orthogonal to each other [26] (see Appendix A for derivations). In addition, the eigenfunctions decay as $t$ approaches infinity [32]. According to (19.8.1) in [31], the parabolic cylinder functions have the asymptotic expression

$$
\left|D_{-\frac{1}{2}-j \omega}(z)\right|=|U(j \omega, z)| \sim\left|e^{-\frac{1}{4} z^{2}} z^{-\frac{1}{2}}\right| .
$$

when $|z| \gg \omega$. This behavior makes the derived eigenfunctions practical as the basis for the eigenfunction expansion. 
4.F.2. Case (F2): $a+d>2, b=0$

In this case, $b=b_{1}=0$, we replace the definition of LCT in (92) with the definition when $b=0$. We obtain

$$
\begin{aligned}
\psi_{\mu_{\mathrm{M}}}^{(1, F 2)}(t) & =\sqrt{d_{1}} e^{j \frac{c_{1} d_{1}}{2} t^{2}} \psi_{\mu_{\mathrm{M}_{0}}}^{(1, F 1)}\left(t d_{1}\right) \\
& =\frac{d_{1}^{j \omega}}{\sqrt{2 \pi}} e^{j \frac{c_{1} d_{1}}{2} t^{2}} t^{-\frac{1}{2}+j \omega}
\end{aligned}
$$

for $t>0$. Note that $d_{1}^{j \omega}$ is the phase factor with unity modulus and we drop it to avoid the phase ambiguity. According to the relations (73-77) and $a d=1$ in this case, we have

$$
\left[\begin{array}{ll}
a_{1} & b_{1} \\
c_{1} & d_{1}
\end{array}\right]=\left[\begin{array}{cc}
a_{1} & 0 \\
\frac{a_{1} c}{a-d} & 1 / a_{1}
\end{array}\right] .
$$

As a result, the eigenfunctions $\psi_{\mathbf{M}}^{(1, F 2)}(t)$ for $t>0$ are

$$
\psi_{\mu_{\mathbf{M}}}^{(1, F 2)}(t)=\frac{1}{\sqrt{2 \pi}} e^{j \frac{c}{2(a-d)} t^{2}} t^{-\frac{1}{2}+j \frac{\mu_{\mathbf{M}}}{d-a}}
$$

and $\psi_{\mu_{\mathbf{M}}}^{(1, F 2)}(t)=0$ for $t \leq 0 . \mu_{\mathbf{M}}$ satisfies (89) and we can simplify them into

$$
\mu_{\mathbf{M}}=(d-a) \omega
$$

by $a d=1$. The second solution in this case is obtained by changing $\psi_{\mathbf{M}_{0}}^{(1, F 1)}(t)$ into $\psi_{\mathbf{M}_{0}}^{(2, F 1)}(t)$ and the derivation remains. We have the following result

$$
\psi_{\mu_{\mathrm{M}}}^{(2, F 2)}(t)=\frac{1}{\sqrt{2 \pi}} e^{j \frac{c}{2(a-d)} t^{2}}(-t)^{-\frac{1}{2}+j \frac{\mu_{\mathrm{M}}}{d-a}}
$$

for $t<0$. It is apparent that (110) is the time-reversed version of (108).

These solutions can be verified easily. The eigenvalues $\mu_{\mathbf{M}}$ and $\lambda_{\mathbf{M}}$ are identical to those derived in (89) and (91) because these expressions are not limited by the condition $b=0$. The only difference is the operator $\mathcal{L}_{\mathbf{M}_{1}}$. Although $\mathcal{L}_{\mathbf{M}_{1}}$ is different, it is still an unitary operator so the derivation steps in (103) can be applied directly without modification. Hence, in Case (F2), the eigenfunctions $\psi_{\mu_{\mathrm{M}}}^{(1, F 2)}(t)$ and $\psi_{\mu_{\mathrm{M}}}^{(2, F 2)}(t)$ are orthogonal to each other.

4.G. Case $(G): a+d<-2$

Our final case, $a+d<-2$, is very similar to Case $(\mathrm{F}), a+d>2$. The matrix $\mathbf{M}$ is decomposed into $[14]$

$$
\left[\begin{array}{ll}
a & b \\
c & d
\end{array}\right]=\left[\begin{array}{ll}
a_{1} & b_{1} \\
c_{1} & d_{1}
\end{array}\right]\left[\begin{array}{cc}
-\sigma^{-1} & 0 \\
0 & -\sigma
\end{array}\right]\left[\begin{array}{ll}
a_{1} & b_{1} \\
c_{1} & d_{1}
\end{array}\right]^{-1}
$$


where

$$
\sigma=\frac{-a-d \pm \sqrt{(a+d)^{2}-4}}{2}, s=\operatorname{sgn}\left(\sigma^{-1}-\sigma\right)
$$

are different from (73) and (74), and other equations are the same as (75-77). We still denote $\mathbf{M}_{0}=\left[-\sigma^{-1}, 0 ; 0,-\sigma\right]$ and $\mathbf{M}_{1}=\left[a_{1}, b_{1} ; c_{1}, d_{1}\right]$ for discussion. The eigenfunctions for $\mathbf{M}_{0}$ are required to be even-symmetric or odd-symmetric. These symmetrical eigenfunctions are obtained by composing $\psi_{\mu_{\mathbf{M}_{0}}}^{(1, F)}(t)$ and $\psi_{\mu_{\mathbf{M}_{0}}}^{(2, F)}(t)$ into even or odd functions, They are

$$
\begin{aligned}
& \psi_{\mu_{\mathbf{M}_{0}}}^{(1, G)}(t)=\frac{1}{\sqrt{2}}\left(\psi_{\mu_{\mathrm{M}_{0}}}^{(1, F)}(t)+\psi_{\mu_{\mathrm{M}_{0}}}^{(2, F)}(t)\right), \\
& \psi_{\mu_{\mathrm{M}_{0}}}^{(2, G)}(t)=\frac{1}{\sqrt{2}}\left(\psi_{\mu_{\mathrm{M}_{0}}}^{(1, F)}(t)-\psi_{\mu_{\mathrm{M}_{0}}}^{(2, F)}(t)\right),
\end{aligned}
$$

where (113) is the even-symmetric eigenfunction and (114) leads to the odd-symmetric eigenfunction.

Following the same steps in (85-89), we have the closed-form expression for $\mu_{\mathbf{M}}$ to be

$$
\mu_{\mathbf{M}}=\operatorname{sgn}\left(\sigma^{-1}-\sigma\right) \omega \sqrt{(a+d)^{2}-4}
$$

The only difference is the term in the sign function due to (112). For the eigenvalues $\lambda_{\mathbf{M}}$, they are divided into two cases based on the symmetry of the eigenfunctions. For the evensymmetric eigenfunctions $\psi_{\mu_{\mathbf{M}_{0}}}^{(1, G)}(t)$, the eigenvalues $\lambda_{\mathbf{M}}^{(1, G)}=\lambda_{\mathbf{M}_{0}}^{(1, G)}$ are

$$
\begin{aligned}
\lambda_{\mathbf{M}}^{(1, G)} & =\frac{\sqrt{-\sigma} \psi_{\mu_{\mathbf{M}_{0}}}^{(1, G)}(-\sigma t)}{\psi_{\mu_{\mathbf{M}_{0}}}^{(1, G)}(t)}=j \frac{\sqrt{\sigma} \psi_{\mu_{\mathbf{M}_{0}}}^{(1, G)}(\sigma t)}{\psi_{\mu_{\mathbf{M}_{0}}}^{(1, G)}(t)} \\
& =j e^{j \omega \log \sigma}=j e^{j \frac{\mu_{\mathbf{M}}}{\operatorname{sgn}\left(\sigma^{-1}-\sigma\right) \sqrt{(a+d)^{2}-4}} \log \sigma} .
\end{aligned}
$$

Note that (116) is similar to (91), except for the constant factor $j$ and the different terms in the sign function. On the other hand, the eigenvalues for the odd eigenfunctions are found to be

$$
\lambda_{\mathbf{M}}^{(2, G)}=-j e^{j \omega \log \sigma}=-j e^{j \frac{\mu_{\mathbf{M}}}{\operatorname{sgn}\left(\sigma^{-1}-\sigma\right) \sqrt{(a+d)^{2}-4}} \log \sigma} .
$$

These eigenvalues are also reasonable. $\mu_{\mathbf{M}}$ are always real because they are similar to Case (F) with very small changes. According to (116) and (117), $\left|\lambda_{\mathbf{M}}^{(1, G)}\right|=\left|\lambda_{\mathbf{M}}^{(2, G)}\right|=1$.

4.G.1. Case (G1): $a+d<-2, b \neq 0$

The eigenfunctions are divided into Case (G1) and Case (G2) because they correspond two different expressions of LCT. According to the previous results, the even solution and the odd solution in Case (F1) are also the eigenfunctions of Case (G1). They are written explicitly 


$$
\begin{aligned}
& \psi_{\mu_{\mathbf{M}}}^{(1, G 1)}(t)=2^{-1 / 2}\left|N_{\omega}\right| e^{j \frac{d-a}{4 b} t^{2}} \\
& {\left[D_{-\frac{1}{2}-j \omega}\left(\frac{e^{j \frac{3 \pi}{4}} t}{\sqrt{\eta}}\right)+D_{-\frac{1}{2}-j \omega}\left(\frac{-e^{j \frac{3 \pi}{4}} t}{\sqrt{\eta}}\right)\right],} \\
& \psi_{\mu_{\mathbf{M}}}^{(2, G 1)}(t)=2^{-1 / 2}\left|N_{\omega}\right| e^{j \frac{d-a}{4 b} t^{2}} \\
& {\left[D_{-\frac{1}{2}-j \omega}\left(\frac{e^{j \frac{3 \pi}{4}} t}{\sqrt{\eta}}\right)-D_{-\frac{1}{2}-j \omega}\left(\frac{-e^{j \frac{3 \pi}{4}} t}{\sqrt{\eta}}\right)\right] .}
\end{aligned}
$$

The even eigenfunctions $\psi_{\mu_{\mathbf{M}}}^{(1, G 1)}(t)$ and the odd eigenfunctions $\psi_{\mu_{\mathbf{M}}}^{(2, G 1)}(t)$ are surely orthogonal to each other. It is because they are derived from the orthogonal functions $\psi_{\mu_{\mathrm{M}_{0}}}^{(1, G)}(t)$ and $\psi_{\mu_{\mathbf{M}_{0}}}^{(2, G)}(t)$.

\section{G.2. Case (G2): $a+d<-2, b=0$}

This case is analogous to Case (F2), except that the eigenfunctions are either even or odd. According the eigenfunctions in Case (F2), (108) and (110), we obtain the two eigenfunctions

$$
\begin{aligned}
& \psi_{\mu_{\mathrm{M}}}^{(1, G 2)}(t)= \begin{cases}\frac{1}{2 \sqrt{\pi}} e^{j \frac{c}{2(a-d)} t^{2}}|t|^{-\frac{1}{2}+j \frac{\mu_{\mathrm{M}}}{d-a}}, & t \neq 0, \\
0 & t=0,\end{cases} \\
& \psi_{\mu_{\mathrm{M}}}^{(2, G 2)}(t)=\frac{1}{2 \sqrt{\pi}} e^{j \frac{c}{2(a-d)} t^{2}}|t|^{-\frac{1}{2}+j \frac{\mu_{\mathrm{M}}}{d-a}} \operatorname{sgn}(t),
\end{aligned}
$$

where $\psi_{\mu_{\mathbf{M}}}^{(1, G 2)}(t)$ is the even solution and $\psi_{\mu_{\mathbf{M}}}^{(2, G 2)}(t)$ is the odd solution. Note that these functions are defined to be zero when $t=0$, in order to avoid the singularity at the origin. These functions are orthogonal to each other due to the orthogonality of $\psi_{\mu_{\mathbf{M}_{0}}}^{(1, G)}(t)$ and $\psi_{\mu_{\mathbf{M}_{0}}}^{(2, G)}(t)$.

\section{Symmetry on LCT Eigenvalues and the Eigenfunctions}

We have already studied the eigenfunctions of the LCT in detail. A summary on the eigenvalues and the eigenfunctions are made in Table 3. In this section, some symmetry about the eigenvalues and the eigenfunctions are going to be discussed.

The eigenvalues $\lambda_{\mathbf{M}}$ in Case (B) and Case (D) are identical when they are expressed in terms of $\mu_{\mathbf{M}}$. The same rule can be applied to Case (C) and Case (E). The eigenvalues for the even/odd solution in Case (C) and Case (E) have an extra phase term $e^{j \pm \pi / 2}$ or $e^{j \pm \operatorname{sgn}(b) \pi / 2}$, which also exists in Case (G1) and Case (G2). The eigenvalues satisfy $\left|\lambda_{\mathbf{M}}\right|=1$ for each case, as discussed in Section 4.

The eigenfunctions also have some resemblance. For $b \neq 0$, they all have the chirp term $e^{j \frac{d-a}{4 b} t^{2}}$, which appears $e^{-j \frac{\tau t^{2}}{2 \sigma^{2}}}$ in Case (A) implicitly and appears in other cases explicitly. These cases are marked with circle $\bigcirc$ in Table 3 . When $b=0$, this chirp term is modified 
into $e^{j \frac{c t^{2}}{2(a-d)}}$ and it exists in Case (F2) and Case (G2) explicitly, and in Case (B) and Case (C) implicitly, where the chirp terms become constant phases due to the delta functions, which can be ignored. The triangle mark $\triangle$ is used to note these cases.

Case (A) and Case (F1), which are denoted by square marks $\square$, also share some similarities. According to (19.13.1) in [31], the parabolic cylinder functions are related to the Hermite Gaussian functions for integer $n$ by

$$
D_{n}(\xi)=2^{-\frac{n}{2}} H_{n}(\xi / \sqrt{2}) e^{-\frac{1}{4} \xi^{2}}, n=0,1,2, \ldots
$$

As a result, it is interpreted that parabolic cylinder functions are generalizations of the Hermite Gaussian functions. The parabolic cylinder functions are more general because the parameters are complex numbers. Besides, it is noted that the parameter $\sqrt{\eta}$ in Case (F1) is similar to the parameter $\sigma$ in Case (A), except that the terms in the square root function are opposite. The eigenfunctions in Case (F1) also have the complex scaling factors $\pm e^{j \frac{3 \pi}{4}}$ in them.

Example 5.1. Here is an example to illustrate the similarity between Case (A) and Case (F1) more closely. Consider the following two transforms

$$
\mathbf{M}_{\alpha}=\left[\begin{array}{cc}
\cos \alpha & \sin \alpha \\
-\sin \alpha & \cos \alpha
\end{array}\right], \mathbf{M}_{\beta}=\left[\begin{array}{ll}
\cosh \beta & \sinh \beta \\
\sinh \beta & \cosh \beta
\end{array}\right]
$$

where $\alpha, \beta \in \mathbb{R}, \mathbf{M}_{\alpha}$ corresponds the fractional Fourier transform and $\mathbf{M}_{\beta}$ is the hyperbolic subgroup $[1,3]$. According to Definition 3.1, The commuting operator for these two cases are

$$
\mathcal{C}_{\mathbf{M}_{\alpha}}=\cos \alpha\left(\mathcal{D}_{t}^{2}-t^{2}\right), \mathcal{C}_{\mathbf{M}_{\beta}}=\cosh \beta\left(\mathcal{D}_{t}^{2}+t^{2}\right)
$$

The factors $\cos \alpha$ and $\cosh \beta$ does not affect the eigenfunctions but the eigenvalues. In quantum mechanics, $\mathcal{C}_{\mathbf{M}_{\alpha}}$ corresponds the harmonic oscillators $[1,6,7,33]$ while $\mathcal{C}_{\mathbf{M}_{\beta}}$ is the inverted oscillators [34-37], or the repulsive oscillators [32,38]. Our eigenfunctions in Case (F1) are exactly the same as the existing results $[32,34]$.

Remark. For Case (F1), an alternative approach was proposed in [17,18]. The authors relate $\mathbf{M}_{\beta}$ with $\mathbf{M}_{\alpha}$ by complex power of the matrices. The resultant eigenfunction for $\mathbf{M}_{\beta}$ is the Hermite Gaussian function with complex scaling factor. Our results are compared with their results using the parabolic cylinder functions. Beginning with (21) in [18], the complex-scaled Hermite Gaussian function can be substituted for parabolic cylinder function, (122), and the eigenfunction is proportional to

$$
D_{n}\left(\frac{t}{e^{j \pi / 4} \sigma}\right) e^{-j \frac{\tau}{2 \sigma^{2}} t^{2}}
$$


Besides, the boundedness of this eigenfunction is checked by

$$
\begin{aligned}
& \left|D_{n}\left(\frac{t}{e^{j \pi / 4} \sigma}\right) e^{-j \frac{\tau}{2 \sigma^{2}} t^{2}}\right|=\left|D_{n}\left(\frac{t}{e^{j \pi / 4} \sigma}\right)\right| \\
& =\left|2^{-n / 2} H_{n}\left(\frac{t}{\sqrt{2} e^{j \pi / 4} \sigma}\right) e^{\frac{j t^{2}}{4 \sigma^{2}}}\right| \\
& =\left|2^{-n / 2} H_{n}\left(\frac{t}{\sqrt{2} e^{j \pi / 4} \sigma}\right)\right| \rightarrow \infty \text { as } t \rightarrow \infty .
\end{aligned}
$$

According to (125), the argument in the parabolic cylinder function, $t /\left(e^{j \frac{\pi}{4}} \sigma\right)$, is identical to our result, $\pm e^{j \frac{3 \pi}{4}} t / \eta$. Besides, the additional chirp term can be simplified as $e^{j \frac{d-a}{4 b} t^{2}}$. The only difference is the index of the parabolic cylinder function. Our eigenfunction evaluates on a straight line on the complex plane, $-\frac{1}{2}-j \omega$; however, the non-negative integer $n$ is required in the result of [18].

Next, the eigenvalues for both results are also compared. Our eigenvalues obey the $\left|\lambda_{\mathbf{M}}\right|=1$ property in each case. But, according to [18], the eigenvalues for the $|a+d|>2$ case are derived to be

$$
\lambda_{n}= \begin{cases}e^{\alpha(n-1 / 2)} & a+d>2, \\ e^{(\alpha-j \pi)(n-1 / 2)} & a+d<-2,\end{cases}
$$

where $\alpha=-\operatorname{arccosh}\left(\left|\frac{a+d}{2}\right|\right) \operatorname{sgn}(b)$. If the parameter $\alpha$ is positive, the eigenvalues for $a+d>2$ grow rapidly as the index $n$ approach to infinity. Due to (104), our results possess bounded eigenfunctions and bounded eigenvalues, which are appropriate for actual computation.

The previous results in [18] and our results are both eigenfunctions of the $a+d>2$ case. The unbounded eigenfunctions in [18] result in non-negative index $n$ while our bounded eigenfunctions compute the index in $-\frac{1}{2}-j \omega$. The same phenomenon sometimes occurs in signal processing, such as the translation operator $\mathcal{T}_{T} x(t)=x(t-T)$. The exponential function and the complex exponential function are trivial eigenfunction of $\mathcal{T}_{T}$ due to

$$
\begin{aligned}
\mathcal{T}_{T} e^{t} & =e^{t-T}=\left(e^{-T}\right) e^{t}, \\
\mathcal{T}_{T} e^{j t} & =e^{j(t-T)}=\left(e^{-j T}\right) e^{j t} .
\end{aligned}
$$

It is shown that for the translation operator $\mathcal{T}_{T}$ with the same parameter $T$, we obtain two distinct sets of eigenvalues and eigenfunctions. In the first set, (128), the real eigenvalue is $e^{-T}$ while the real eigenfunction is $e^{t}$, implying the magnitude of the eigenvalue is not always unity and the eigenfunction grows unbounded as $t$ approaches to infinity. On the other hand, the second set of solutions has complex eigenvalue $e^{-j T}$ and complex eigenfunction $e^{j t}$. We have unity magnitude of eigenvalue $\left(\left|e^{-j T}\right|=1\right)$ and bounded eigenfunction $\left(\left|e^{j t}\right|=1\right)$.

It is observed that a small change on the exponential function changes the boundedness and the resultant eigenvalue is confined in the unit circle, as indicated in (129). The previous results in [18] is similar to (128); our eigenfunctions for $|a+d|>2$ resemble (129) more. 


\section{Application: Eigen-Decomposition of the LCT}

A major application is the the discrete LCT. Apart from sampling the integral definition (1), the LCT can be implemented by the signal expansion on the eigenspace. Our method features perfect reconstruction when taking the inverse LCT.

An illustrative example is given in Fig. 2. Assume that the input signal is the rectangular function

$$
x(t)=\frac{1}{\sqrt{2}} \operatorname{rect}\left(\frac{t}{2}\right)= \begin{cases}1 / \sqrt{2} & |t|<1, \\ 0 & \text { otherwise. }\end{cases}
$$

The signal plot is shown in Fig. 2(b). The Wigner distribution function (WDF) of $x(t)$ is also computed in Fig. 2(a), where the energy distribution is marked by a dashed rectangle. Based on the complete and orthonormal eigenfunctions, the LCT can be implemented as the following three steps:

1. Eigenfunction expansion (projection): Given $x(t)$, the goal is to evaluate the coefficients $a_{\mu_{\mathrm{M}}}$ such that

$$
x(t)=\sum_{\mu_{\mathbf{M}}} a_{\mu_{\mathbf{M}}} \psi_{\mu_{\mathrm{M}}}(t) .
$$

Due to the orthogonal property of the eigenfunctions, the coefficients are obtained by taking the inner products of $\psi_{\mu_{\mathrm{M}}}(t)$ and $x(t)$ :

$$
a_{\mu_{\mathrm{M}}}=\left\langle\psi_{\mu_{\mathrm{M}}}(t), x(t)\right\rangle=\int_{-\infty}^{\infty} \psi_{\mu_{\mathrm{M}}}^{*}(t) x(t) \mathrm{d} t .
$$

(132) is interpreted as the eigenfunction analysis equation for the input signal $x(t)$, which projects $x(t)$ onto the eigenspace to have coefficients $a_{\mu_{\mathrm{M}}}$.

In the example, we select $\mathbf{M}=[1,0.4 ;-4.75,-0.9]$ and then compute the eigenfunctions based on our closed-form eigenfunctions, as shown in Fig. 2(c,d), where the real parts, the imaginary parts, and the magnitudes are all drawn on the plots.

2. Multiply $\lambda_{\mathbf{M}}$ : Taking the LCT on both sides of (131) yields

$$
X_{\mathbf{M}}(t)=\sum_{\mu_{\mathbf{M}}} \lambda_{\mathbf{M}} a_{\mu_{\mathbf{M}}} \psi_{\mu_{\mathbf{M}}}(t),
$$

which is depicted in the bottom of Fig. 2. In this step, $\lambda_{\mathbf{M}} a_{\mu_{\mathbf{M}}}$ is to be computed. Note that the relationship between $\lambda_{\mathbf{M}}$ and $\mu_{\mathbf{M}}$ was studied completely in Section 4 . We can utilize these relations for any $\mu_{\mathbf{M}}$.

The eigenvalues $\lambda_{\mathbf{M}}$ can be rewritten as its magnitude and phase by $\left|\lambda_{\mathbf{M}}\right| e^{j \angle \lambda_{\mathbf{M}}}$, where $\angle \lambda_{\mathbf{M}}$ indicates the phase of $\lambda_{\mathbf{M}}$. Substituting this alternative expression into (133) and applying Property $4\left(\left|\lambda_{\mathbf{M}}\right|=1\right)$ yield $a_{\mu_{\mathbf{M}}}\left(e^{j \angle \lambda_{\mathbf{M}}} \psi_{\mu_{\mathbf{M}}}(t)\right)$ for the summand. It is 
evident that this step is equivalent to appending additional phase factors $e^{j \angle \lambda_{\mathrm{M}}}$ to the eigenfunctions.

3. Eigenfunction combination: The final step is to sum up $\lambda_{\mathbf{M}} a_{\mu_{\mathbf{M}}} \psi_{\mu_{\mathbf{M}}}(t)$ over all $\mu_{\mathbf{M}}$, as (133) states. In Fig. 2(g,h), $\lambda_{\mathbf{M}} \psi_{\mu_{\mathbf{M}}}(t)$ is plotted. By comparing (c) and (g), it is observed that $\psi_{\mu_{\mathbf{M}}}(t)$ and $\lambda_{\mathbf{M}} \psi_{\mu_{\mathbf{M}}}(t)$ are identical in terms of magnitude so does (d) and (h). These observations justify Property 4, which claims $\left|\lambda_{\mathbf{M}}\right|=1$.

After the three-step implementation, the resultant LCT $X_{\mathbf{M}}(t)$ is shown in Fig. 2(f), where the magnitude information is plotted in solid curve. The WDF of $X_{\mathbf{M}}(t)$ is also given in Fig. 2 (e) for clarity. The energy distribution is now twisted into the parallelogram, which is marked in dashed lines, by the affine transformation with parameter $a, b, c$ and $d$. This illustrative example proves that the eigenfunctions can be applied to the LCT implementation.

The LCT via eigenfunctions has the following features other than the existing method $[39,40]$. The eigenfunctions are proved to be complete and orthogonal. Hence, taking the inverse LCT operation $\mathcal{L}_{\mathbf{M}^{-1}}$ follows the three-steps implementation as long as the eigenvalues are modified into $\lambda_{\mathbf{M}}^{-1}$. Perfect recovery of the signal is ensured under our implementation scheme.

In addition, signal expansion provides a tool for signal processing under these novel eigenfunctions. The eigenfunctions are physically meaningful in quantum mechanics and optics. For instance, these eigenfunctions establish a orthonormal basis for studying the self-imaging phenomenon in lossless media. These eigenfunctions also act as the wavefunctions in the quantum system related to the LCT. In signal processing, after LCT operation, the transformed output of the eigenfunction remains the same form with an additional eigenvalue phase factor. The expansion coefficients $a_{\mu_{\mathrm{M}}}$ are able to be applied to signal analysis, signal estimation, and signal modeling.

\section{Conclusion}

In this paper, the commuting operator $\mathcal{C}_{\mathbf{M}}$, which commutes with LCT operator $\mathcal{L}_{\mathrm{M}}$, was presented. It is shown that this operator and the LCT operator share the same eigenfunctions but have different eigenvalues. The analysis using the commuting operator unifies previous work and enables it to be examined in an uniform manner; it also extends previous research by finding the closed-form and bounded expression for the eigenvalues and the eigenfunctions in the cases of $|a+d|>2$ and $a+d= \pm 2, b \neq 0$. Our work is summarized in Table 3 .

In the future, the discrete LCT with perfect reconstruction property can be efficiently calculated by the eigenfunctions. Based on the commuting operator $\mathcal{C}_{\mathbf{M}}$, and the closed-form expressions of $\lambda_{\mathbf{M}}$, we can achieve discrete implementation of FrFT, scaling operation, chirp convolution, chirp multiplication, or combinations of these operations, which are very useful 
in digital signal processing.

\section{Appendix A: Inner products between special functions}

The inner products between some special functions throughout the paper are derived in this appendix for clarity. The first one is $\langle\delta(t-\alpha), \delta(t-\beta)\rangle$. Recall that the Dirac delta function is defined as the (inverse) Fourier transform of the constant function as follows

$$
\delta(t-\alpha)=\frac{1}{2 \pi} \int_{-\infty}^{\infty} e^{ \pm j \omega(t-\alpha)} \mathrm{d} \omega
$$

Taking the inner product between two deltas and applying (A1) yield

$$
\langle\delta(t-\alpha), \delta(t-\beta)\rangle=\frac{1}{4 \pi^{2}} \int_{-\infty}^{\infty} \int_{-\infty}^{\infty} \int_{-\infty}^{\infty} e^{j \omega_{1}(t-\alpha)} e^{j \omega_{2}(t-\beta)} \mathrm{d} \omega_{1} \mathrm{~d} \omega_{2} \mathrm{~d} t .
$$

Integrating the variable $t$ first gives us

$$
\langle\delta(t-\alpha), \delta(t-\beta)\rangle=\frac{1}{2 \pi} \int_{-\infty}^{\infty} \int_{-\infty}^{\infty} e^{-j \omega_{1} \alpha} e^{-j \omega_{2} \beta} \delta\left(\omega_{1}+\omega_{2}\right) \mathrm{d} \omega_{1} \mathrm{~d} \omega_{2} .
$$

Note that the sifting property of the delta function, $\int_{-\infty}^{\infty} f(t) \delta\left(t-t_{0}\right) \mathrm{d} t=f\left(t_{0}\right)$, can be applied to the integrals. Letting $\omega_{1}=-\omega_{2}$ and eliminating the integral with respect to $\omega_{1}$ lead to the following result

$$
\langle\delta(t-\alpha), \delta(t-\beta)\rangle=\frac{1}{2 \pi} \int_{-\infty}^{\infty} e^{j \omega_{2}(\alpha-\beta)} \mathrm{d} \omega_{2}=\delta(\alpha-\beta) .
$$

Next, the orthogonal property between two complex exponentials is verified. By definition,

$$
\left\langle\frac{1}{\sqrt{2 \pi}} e^{j \omega_{1} t}, \frac{1}{\sqrt{2 \pi}} e^{j \omega_{2} t}\right\rangle=\frac{1}{2 \pi} \int_{-\infty}^{\infty} e^{-j\left(\omega_{1}-\omega_{2}\right) t} \mathrm{~d} t
$$

According to (A1), we have the orthogonal property $\left\langle e^{j \omega_{1} t} / \sqrt{2 \pi}, e^{j \omega_{2} t} / \sqrt{2 \pi}\right\rangle=\delta\left(\omega_{1}-\omega_{2}\right)$.

To prove the orthogonal property of the scale-invariant function in (83), taking the inner product of $h_{\omega}(t)$ as defined in $(80)$,

$$
\left\langle h_{\omega_{1}}(t), h_{\omega_{2}}(t)\right\rangle=\frac{1}{2 \pi} \int_{0}^{\infty} t^{-1-j\left(\omega_{1}-\omega_{2}\right)} \mathrm{d} t=\frac{1}{2 \pi} \int_{0}^{\infty} e^{-j\left(\omega_{1}-\omega_{2}\right) \log t} t^{-1} \mathrm{~d} t .
$$

Making change of variables $\tau=\log t$ results in $\mathrm{d} \tau=t^{-1} \mathrm{~d} t$ and

$$
\left\langle h_{\omega_{1}}(t), h_{\omega_{2}}(t)\right\rangle=\frac{1}{2 \pi} \int_{-\infty}^{\infty} e^{-j\left(\omega_{1}-\omega_{2}\right) \tau} \mathrm{d} \tau
$$

Applying the orthogonal property of the complex exponential functions to (A7), we obtain $\left\langle h_{\omega_{1}}(t), h_{\omega_{2}}(t)\right\rangle=\delta\left(\omega_{1}-\omega_{2}\right)$. 


\section{Appendix B: Eigenvalues of Case (C) in [14]}

We examine the derivation steps (60) to (61) in [14]. Substituting (61) into (60) in [14] and computing the terms in the brackets we have

$$
\begin{aligned}
& O_{F}^{(-1,0, c,-1)}\left(\phi_{C}^{c, h}(t)\right)=j e^{-j c u^{2} / 2} \\
& \quad\left[\delta\left(-u-\sqrt{\frac{4 n \pi}{|c|}+h}\right)+\delta\left(-u+\sqrt{\frac{4 n \pi}{|c|}+h}\right)\right] .
\end{aligned}
$$

According to $\delta(-t)=\delta(t)$, we obtain

$$
\begin{aligned}
& O_{F}^{(-1,0, c,-1)}\left(\phi_{C}^{c, h}(t)\right)=j e^{-j c u^{2} / 2} \\
& \quad\left[\delta\left(u+\sqrt{\frac{4 n \pi}{|c|}+h}\right)+\delta\left(u-\sqrt{\frac{4 n \pi}{|c|}+h}\right)\right] .
\end{aligned}
$$

From the sifting property of the delta functions, $x(t) \delta\left(t-t_{0}\right)=x\left(t_{0}\right) \delta\left(t-t_{0}\right)$, we obtain the eigenvalues to be $j e^{-j c h / 2}$. The modified result is then exactly identical to our newly derived result.

\section{Acknowledgments}

This work was supported by the National Science Council, R.O.C., under Contract 98-2221E-002-077-MY3.

\section{References}

1. H. M. Ozaktas, Z. Zalevsky, and M. A. Kutay, The Fractional Fourier Transform with Applications in Optics and Signal Processing (Wiley, 2001).

2. S. Abe and J. T. Sheridan, "Optical operations on wave functions as the Abelian subgroups of the special affine Fourier transformation," Opt. Lett. 19, 1801-1803 (1994).

3. K. B. Wolf, Integral Transforms in Science and Engineering (Plenum Publ. Corp., 1979).

4. D. F. James and G. S. Agarwal, "The generalized Fresnel transform and its application to optics," Optics Communications 126, 207212 (1996).

5. L. M. Bernardo, "ABCD matrix formalism of fractional Fourier optics," Optical Eng. 35, 732-740 (1996).

6. D. J. Griffiths, Introduction to Quantum Mechanics (Pearson Prentice Hall, 2005).

7. V. Namias, "The Fractional Order Fourier Transform and its Application to Quantum Mechanics," J. Inst. Maths Applics 25, 241-265 (1980).

8. H. M. Ozaktas and D. Mendlovic, "Fractional Fourier optics," J. Opt. Soc. Am. A 12, 743-751 (1995). 
9. H. T. Yura and S. G. Hanson, "Optical beam wave propagation through complex optical systems," J. Opt. Soc. Am. A 4, 1931-1948 (1987).

10. D. Li, D. P. Kelly, R. Kirner and J. T. Sheridan, "Speckle orientation in paraxial optical systems," Appl. Opt. 51, A1-A10 (2012).

11. D. P. Kelly, J. J. Healy, B. M. Hennelly and J. T. Sheridan, "Quantifying the 2.5D imaging performance of digital holographic systems," J. Europ. Opt. Soc. Rap. Public. 110346 (2011).

12. S.-C. Pei and J.-J. Ding, "Fractional Fourier transform, Wigner distribution, and filter design for stationary and nonstationary random processes," IEEE Trans. Signal Process. 58, 4079-4092 (2010).

13. D. Mustard, "The fractional Fourier transform and the Wigner distribution," J.Austral. Math. Soc. Ser. B 38, 209-219 (1996).

14. S.-C. Pei and J.-J. Ding, "Eigenfunctions of Linear Canonical Transform," IEEE Trans. Signal Process. 50, 11-26 (2002).

15. J. J. Ding, "Research of fractional Fourier transform and linear canonical transform," Ph.D. thesis, National Taiwan University (2001).

16. G. W. Wornell, Signal Processing with Fractals: A Wavelet-Based Approach (PrenticeHall, 1996).

17. T. Alieva and M. J. Bastiaans, "Properties of eigenfunctions of the canonical integral transform," in "Proc. IEEE EURASIP Workshop on Nonlinear Signal and Image Processing," , vol. 2 (1999), vol. 2, pp. 585-587.

18. A. Serbes, S. Aldrmaz, and L. Durak-Ata, "Eigenfunctions of the linear canonical transform," in "Proc. of 20th Signal Processing and Communications Applications Conference (SIU)," (2012), pp. 1-4.

19. A. Koç, H. M. Ozaktas, C. Candan, and M. A. Kutay, "Digital Computation of Linear Canonical Transforms," IEEE Trans. Signal Process. 56, 2383-2394 (2008).

20. J. W. Goodman, Introduction to Fourier Optics (Roberts and Company Publishers, 2005).

21. C. Candan, M. A. Kutay, and H. M. Ozaktas, "The Discrete Fractional Fourier Transform," IEEE Trans. Signal Process. 48, 1329-1337 (2000).

22. C. Candan, "On Higher Order Approximations for Hermite-Gaussian Functions and Discrete Fractional Fourier Transforms," IEEE Signal Process. Lett. 14, 699-702 (2007).

23. H. Kogelnik and T. Li, "Laser Beams and Resonators," Appl. Opt. 5, 1550-1567 (1966).

24. A. E. Siegman, Lasers (University Science Books, 1986).

25. R. N. Bracewell, The Fourier Transform and Its Applications (McGrawHill, 2002), 3rd ed.

26. L. Cohen, "The Scale Representation," IEEE Trans. Signal Process. 41, 3275-3292 
(1993).

27. R. G. Baraniuk and D. Jones, "Unitary equivalence: a new twist on signal processing," IEEE Trans. Signal Process. 43, 2269-2282 (1995).

28. R. G. Baraniuk, "Signal transform covariant to sccale changes," Electronics Letters 29, 1675-1676 (1993).

29. M. Izzetoğlu, B. Onaral, P. Chitrapu, and N. Bilgutay, "Discrete Time Processing of Linear Scale Invariant Signals and Systems," Proc. SPIE 4116, 110-118 (2000).

30. F. Oberhettinger, Tables of Mellin Transforms (Springer-Verlag, 1974).

31. M. Abramowitz and I. A. Stegun, eds., Handbook of Mathematical Functions With Formulas, Graphs, and Mathematical Tables (1965).

32. C. A. Muñoz, J. Rueda-Paz, and K. B. Wolf, "Discrete repulsive oscillator wavefunctions," J. Phys. A: Math. Theor. 42 (2009).

33. L. Barker, C. Candan, T. Hakioglu, M. A. Kutay, and H. M. Ozaktas, "The discrete harmonic oscillator, harper's equation, and the discrete fractional fourier transform," J. Phys. A: Math. Gen. 33, 2209-2222 (2000).

34. E. G. Kalnins and W. Miller, "Lie theory and separation of variables. 5. The equations $i U_{t}+U_{x x}=0$ and $i U_{t}+U_{x x} c / x^{2} U=0$," J. Math. Phys. 15, 1728-1737 (1974).

35. G. Barton, "Quantum Mechanics of the Inverted Oscillator Potential," Annals of Physics 166, 322-363 (1986).

36. S. Tarzi, "The inverted harmonic oscillator: some statistical properties," J. Phys. A: Math. Gen. 21, 3105-3111 (1988).

37. C. Yuce, A. Kilic, and A. Coruh, "Inverted oscillator," Phys. Scr. 74, 114-116 (2006).

38. P. G. L. Leach, "Sl(3,R) and the repulsive oscillator," J. Phys. A: Math. Gen. 13, 1991-2000 (1980).

39. B. M. Hennelly and J. T. Sheridan, "Fast numerical algorithm for the linear canonical transform," J. Opt. Soc. Am. A 22, 928-937 (2005).

40. S.-C. Pei and Y.-C. Lai, "Discrete linear canonical transforms based on dilated Hermite functions," J. Opt. Soc. Am. A 28, 1695-1708 (2011). 


\section{List of Figures}

1 Different cases for the eigenfunctions of the LCT, modified from [14]. . . . .

2 The illustration of the eigen-expansion of the LCT. (b) The input signal $x(t)$ and (a) its Wigner distribution function (WDF) $\mathrm{WDF}_{x}(t, f)$. For $\mathbf{M}=[1,0.4 ;-4.75,-0.9]$, the first eigenfunction is listed in (c) while (d) shows the second eigenfunction. The resultant LCT $X_{\mathbf{M}}(t)$ is plotted in (f). (e) stands for the WDF of $X_{\mathbf{M}}(t)$. (g) and (h) indicate the eigenfunctions multiplied by the eigenvalues $\lambda_{\mathbf{M}}=\left|\lambda_{\mathbf{M}}\right| e^{j \angle \lambda_{\mathbf{M}}}=e^{j \angle \lambda_{\mathbf{M}}}$, respectively. In (g) and $(\mathrm{h})$, it is obvious that the magnitudes remain unchanged, the additional phase terms are appended, and the real as well as imaginary parts differ from those in $(\mathrm{c})$ and $(\mathrm{d}) . \ldots \ldots \ldots \ldots$ 


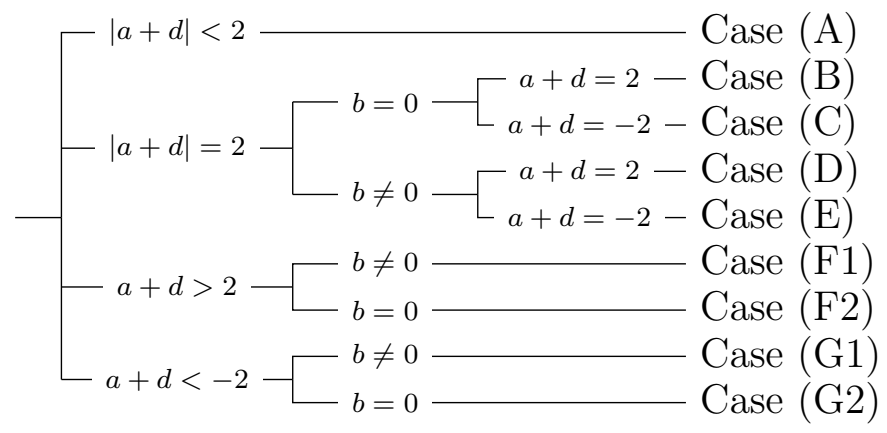

Fig. 1. Different cases for the eigenfunctions of the LCT, modified from [14]. 


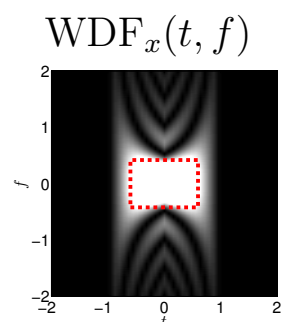

(a)

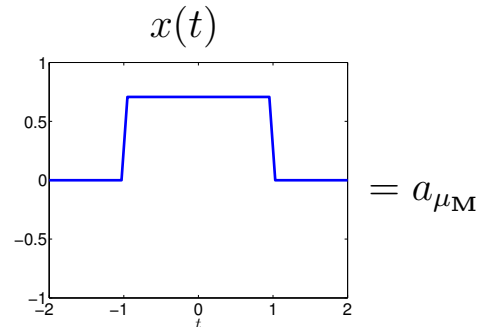

(b)

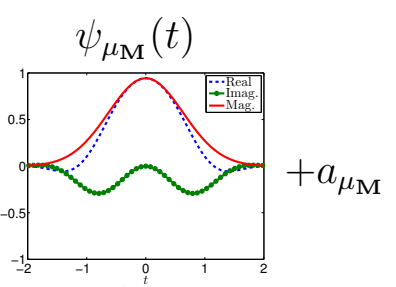

(c)

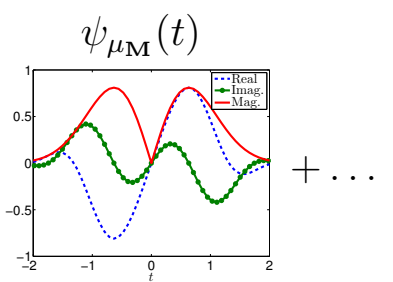

(d)

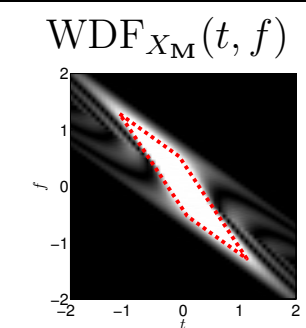

(e)

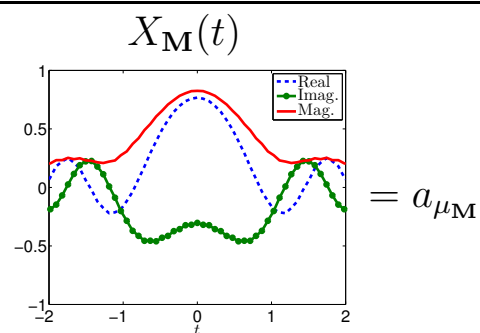

(f)

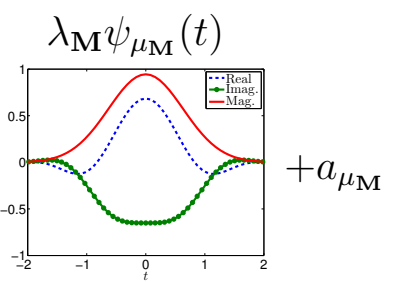

(g)

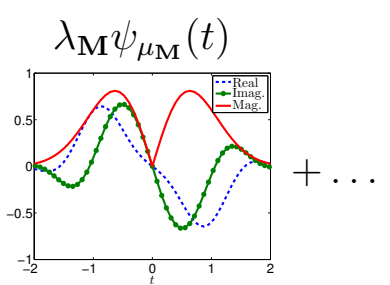

(h)

Fig. 2. The illustration of the eigen-expansion of the LCT. (b) The input signal $x(t)$ and (a) its Wigner distribution function (WDF) $\mathrm{WDF}_{x}(t, f)$. For $\mathbf{M}=[1,0.4 ;-4.75,-0.9]$, the first eigenfunction is listed in (c) while (d) shows the second eigenfunction. The resultant LCT $X_{\mathbf{M}}(t)$ is plotted in (f). (e) stands for the WDF of $X_{\mathbf{M}}(t)$. (g) and (h) indicate the eigenfunctions multiplied by the eigenvalues $\lambda_{\mathbf{M}}=\left|\lambda_{\mathbf{M}}\right| e^{j \angle \lambda_{\mathbf{M}}}=e^{j \angle \lambda_{\mathbf{M}}}$, respectively. In (g) and (h), it is obvious that the magnitudes remain unchanged, the additional phase terms are appended, and the real as well as imaginary parts differ from those in (c) and $(\mathrm{d})$. 


\section{List of Tables}

1 Summary on the eigenfunctions and the eigenvalues [14] . . . . . . . . 35

2 The commuting operators for some special cases of the LCT. . . . . . . . 36

3 Summary on LCT Eigenfunctions and Eigenvalues . . . . . . . . . . . . . 37 
Table 1. Summary on the eigenfunctions and the eigenvalues [14]

\begin{tabular}{|c|c|c|c|}
\hline Case & Eigenfunctions $\psi(t)$ & Eigenvalues & Parameters \\
\hline (A) $|a+d|<2$ & $\left(\frac{1}{\sqrt{\pi} \sigma 2^{n} n !}\right)^{1 / 2} H_{n}\left(\frac{t}{\sigma}\right) e^{-\frac{1+j \tau}{2 \sigma^{2}} t^{2}}$ & $e^{-j\left(n+\frac{1}{2}\right) \alpha}$ & $\begin{array}{l}\sigma^{2}=\frac{2|b|}{\sqrt{4-(a+d)^{2}}}, \\
\tau=\frac{\operatorname{sgn}(b)(a-d)}{\sqrt{4-(a+d)^{2}}}, \\
\alpha=\cos ^{-1} \frac{a+d}{2} \\
\sin ^{-1} \frac{\operatorname{sgn}(b) \sqrt{4-(a+d)^{2}}}{2} .\end{array}$ \\
\hline $\begin{array}{l}\text { (B) } a+d=2 \\
b=0\end{array}$ & $\begin{array}{l}E^{-\frac{1}{2}}\left(\sum_{n=0}^{\infty} A_{n} \delta\left(t-\sqrt{\frac{4 n \pi}{|c|}+h}\right)\right. \\
\left.\quad+\sum_{m=0}^{\infty} B_{m} \delta\left(t+\sqrt{\frac{4 m \pi}{|c|}+h}\right)\right)\end{array}$ & $e^{j c h / 2}$ & $\begin{array}{l}0 \leq h \leq \frac{4 \pi}{|c|}, \quad E= \\
\sum_{n=0}^{\infty}\left(\left|A_{n}\right|^{2}+\left|B_{n}\right|^{2}\right)\end{array}$ \\
\hline $\begin{array}{l}\text { (C) } a+d=-2 \\
b=0\end{array}$ & $\begin{array}{l}\text { The same case as B, except for } A_{n}= \pm B_{n} \text { for all } \\
n \text {. }\end{array}$ & $\pm j e^{-j c h / 2}$ & Explained in (B1) and (B2) \\
\hline $\begin{array}{l}\text { (D) } a+d=2 \\
b \neq 0\end{array}$ & $\sqrt{\frac{1}{j 2 \pi \rho}} e^{j \frac{d-a}{4 b} t^{2}} \int_{-\infty}^{\infty} e^{j \frac{(t-\xi)^{2}}{2 \rho}} g(\xi) \mathrm{d} \xi$ & $e^{-j b h / 2}$ & $\begin{array}{l}g(t) \\
\sqrt{\frac{1}{2 \pi S}}\left[\sum_{n=0}^{\infty} C_{n} e^{j t \sqrt{\frac{4 n \pi}{b \mid}+h}}=\right. \\
\quad+\sum_{m=0}^{\infty} D_{m} e^{-j t \sqrt{\frac{4 m \pi}{|b|}+h}} \\
0 \leq h<\frac{4 \pi}{|b|}, \quad S= \\
\sum_{n=0}^{\infty}\left(\left|C_{n}\right|^{2}+\left|D_{n}\right|^{2}\right)\end{array}$ \\
\hline $\begin{array}{l}\text { (E) } a+d=-2, \\
b \neq 0\end{array}$ & The same as case D, except for $C_{n}= \pm D_{n}$. & $\pm j e^{-j b h / 2}$ & \\
\hline (F) $a+d>2$ & $\frac{1}{\sqrt{j 2 \pi \eta}} e^{j \frac{\tau}{2} t^{2}} \int_{-\infty}^{\infty} e^{\frac{j}{2 \eta}(t-\xi)^{2}} h(\xi) \mathrm{d} \xi$ & $\lambda$ & $\begin{array}{l}\sqrt{\sigma} h(\sigma t)=\lambda h(t), \\
\tau=\frac{-2 s c}{s(d-a)+\sqrt{(a+d)^{2}-4}}, \eta= \\
\frac{s b}{\sqrt{(a+d)^{2}-4}}, \\
\sigma=\frac{a+d \pm \sqrt{(a+d)^{2}-4}}{2}, s= \\
\operatorname{sgn}\left(\sigma-\sigma^{-1}\right) .\end{array}$ \\
\hline (G) $a+d<-2$ & $\begin{array}{l}\text { The same as case } \mathrm{F} \text {, except for } h(t)= \pm h(-t) \\
\text { must be satisfied and } s=\operatorname{sgn}\left(\sigma^{-1}-\sigma\right)\end{array}$ & $\lambda$ & \\
\hline
\end{tabular}


Table 2. The commuting operators for some special cases of the LCT.

\begin{tabular}{cc}
\hline Operation & Commuting operator \\
\hline Scaling & $t \mathcal{D}_{t}+\mathcal{D}_{t} t$ \\
Chirp convolution & $\mathcal{D}_{t}^{2}$ \\
Chirp multiplication & $t^{2}$ \\
(fractional) Fourier transform & $\mathcal{D}_{t}^{2}-t^{2}$ \\
hyperbolic & $\mathcal{D}_{t}^{2}+t^{2}$ \\
\hline
\end{tabular}


Table 3. Summary on LCT Eigenfunctions and Eigenvalues

\begin{tabular}{|c|c|c|c|}
\hline Case & Eigenfunctions $\psi_{\mu_{\mathbf{M}}}(t)$ & Eigenvalues $\lambda_{\mathbf{M}}$ & Parameters \\
\hline $\begin{array}{l}\text { (A) }|a+d|< \\
\square 2, b \neq 0\end{array}$ & $\left(\frac{1}{\sqrt{\pi} \sigma 2^{n} n !}\right)^{1 / 2} H_{n}\left(\frac{t}{\sigma}\right) e^{-\frac{1+j \tau}{2 \sigma^{2}} t^{2}}$ & $e^{-j\left(n+\frac{1}{2}\right) \alpha}$ & $\begin{array}{l}\sigma^{2}=\frac{2|b|}{\sqrt{4-(a+d)^{2}}}, \\
\tau=\frac{\operatorname{sgn}(b)(a-d)}{\sqrt{4-(a+d)^{2}}}, \\
\alpha=\cos ^{-1} \frac{a+d}{2} \\
\sin ^{-1} \frac{\operatorname{sgn}(b) \sqrt{4-(a+d)^{2}}}{2} .\end{array}$ \\
\hline$\triangle \begin{array}{l}(\mathrm{B}) a+d=2 \\
b=0\end{array}$ & $\delta\left(t \pm \sqrt{\mu_{\mathbf{M}} / c}\right)$ & $e^{j \frac{1}{2} \mu_{\mathbf{M}}}$ & $\mu_{\mathrm{M}} / c \geq 0$ \\
\hline $\begin{array}{l}(\mathrm{C}) a+d= \\
-2, b=0\end{array}$ & $\frac{1}{\sqrt{2}}\left(\delta\left(t+\sqrt{\mu_{\mathbf{M}} / c}\right) \pm \delta\left(t-\sqrt{\mu_{\mathrm{M}} / c}\right)\right)$ & $e^{-\frac{j}{2}\left(\mu_{\mathbf{M}} \mp \pi\right)}$ & $\mu_{\mathrm{M}} / c \geq 0$ \\
\hline $\begin{array}{l}\text { (D) } a+d=2 \\
b \neq 0\end{array}$ & $\frac{1}{\sqrt{2 \pi}} e^{j \frac{d-a}{4 b} t^{2}} e^{ \pm j t \sqrt{-\mu_{\mathbf{M}} / b}}$ & $e^{j \frac{1}{2} \mu_{\mathbf{M}}}$ & $\mu_{\mathrm{M}} / b \leq 0$ \\
\hline $\begin{array}{l}(\mathrm{E}) a+d= \\
-2, b \neq 0\end{array}$ & $\frac{1}{\sqrt{\pi}} e^{j \frac{d-a}{4 b} t^{2}}\left\{\begin{array}{l}\cos \\
\sin \end{array}\right\}\left(\sqrt{\frac{-\mu_{\mathbf{M}}}{b}} t\right)$ & $e^{-\frac{j}{2}\left(\mu_{\mathbf{M}} \pm \operatorname{sgn}(b) \pi\right)}$ & $\mu_{\mathrm{M}} / b \leq 0$ \\
\hline $\begin{array}{l}\text { (F1) } a+d>2 \\
\square \quad b \neq 0\end{array}$ & $\left|N_{\omega}\right| D_{-\frac{1}{2}-j \omega}\left(\frac{ \pm e^{j \frac{3 \pi}{4}} t}{\sqrt{\eta}}\right) e^{j \frac{d-a}{4 b} t^{2}}$ & $e^{j \omega \log \sigma}$ & $\begin{array}{l}\eta=\frac{s b}{\sqrt{(a+d)^{2}-4}}, \quad \sigma= \\
\frac{a+d \pm \sqrt{(a+d)^{2}-4}}{2}, \\
s=\operatorname{sgn}\left(\sigma-\sigma^{-1}\right), N_{\omega} \text { is de- } \\
\text { fined in }(99), \\
\mu_{\mathrm{M}}=s \omega \sqrt{(a+d)^{2}-4}, \\
\omega \in \mathbb{R} .\end{array}$ \\
\hline $\begin{array}{l}(\mathrm{F} 2) a+d>2 \\
b=0\end{array}$ & $\frac{1}{\sqrt{2 \pi}} e^{j \frac{c}{2(a-d)} t^{2}}\left\{\begin{array}{c}t \\
-t\end{array}\right\}^{-\frac{1}{2}+j \frac{\mu_{\mathbf{M}}}{d-a}}$ for $\left\{\begin{array}{l}t>0 \\
t<0\end{array}\right\}$ & $e^{j \omega \log \sigma}$ & $\mu_{\mathrm{M}}=(d-a) \omega, \omega \in \mathbb{R}$ \\
\hline $\begin{array}{l}(\mathrm{G} 1) a+d< \\
-2, b \neq 0\end{array}$ & $\begin{array}{l}2^{-1 / 2}\left|N_{\omega}\right| e^{j \frac{d-a}{4 b} t^{2}} \\
{\left[D_{-\frac{1}{2}-j \omega}\left(\frac{e^{j \frac{3 \pi}{4}}}{\sqrt{\eta}} t\right) \pm D_{-\frac{1}{2}-j \omega}\left(\frac{-e^{j \frac{3 \pi}{4}}}{\sqrt{\eta}} t\right)\right]}\end{array}$ & $\pm j e^{j \omega \log \sigma}$ & $\begin{array}{l}\eta=\frac{s b}{\sqrt{(a+d)^{2}-4}}, \quad \sigma= \\
\frac{-a-d \pm \sqrt{(a+d)^{2}-4}}{2} \\
s=\operatorname{sgn}\left(\sigma^{-1}-\sigma\right), N_{\omega} \text { is de- } \\
\text { fined in }(99), \\
\mu_{\mathrm{M}}=s \omega \sqrt{(a+d)^{2}-4}, \\
\omega \in \mathbb{R} .\end{array}$ \\
\hline $\begin{array}{l}(\mathrm{G} 2) a+d< \\
-2, b=0\end{array}$ & $\frac{1}{2 \sqrt{\pi}} e^{j \frac{c}{2(a-d)} t^{2}}|t|^{-\frac{1}{2}+j \frac{\mu_{\mathbf{M}}}{d-a}}\left\{\begin{array}{c}1 \\
\operatorname{sgn}(t)\end{array}\right\}$ & $\pm j e^{j \omega \log \sigma}$ & $\mu_{\mathrm{M}}=(d-a) \omega, \omega \in \mathbb{R}$ \\
\hline
\end{tabular}

